\title{
Conducting polymers/zinc oxide-based photocatalysts for environmental remediation: a review
}

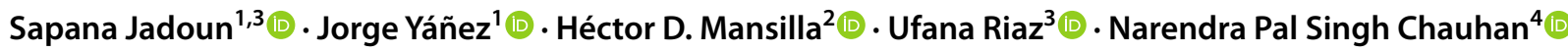

Received: 18 May 2021 / Accepted: 17 January 2022 / Published online: 19 February 2022

(c) The Author(s), under exclusive licence to Springer Nature Switzerland AG 2022

\begin{abstract}
The accessibility to clean water is essential for humans, yet nearly 250 million people die yearly due to contamination by cholera, dysentery, arsenicosis, hepatitis A, polio, typhoid fever, schistosomiasis, malaria, and lead poisoning, according to the World Health Organization. Therefore, advanced materials and techniques are needed to remove contaminants. Here, we review nanohybrids combining conducting polymers and zinc oxide for the photocatalytic purification of waters, with focus on in situ polymerization, template synthesis, sol-gel method, and mixing of semiconductors. Advantages include less corrosion of zinc oxide, less charge recombination and more visible light absorption, up to 53\%.
\end{abstract}

Keywords Conducting polymers $\cdot$ Zinc oxide $\cdot$ Photocatalysis $\cdot$ Environmental remediation $\cdot$ Polyaniline $\cdot$ Poly $(o-$ phenylenediamine)

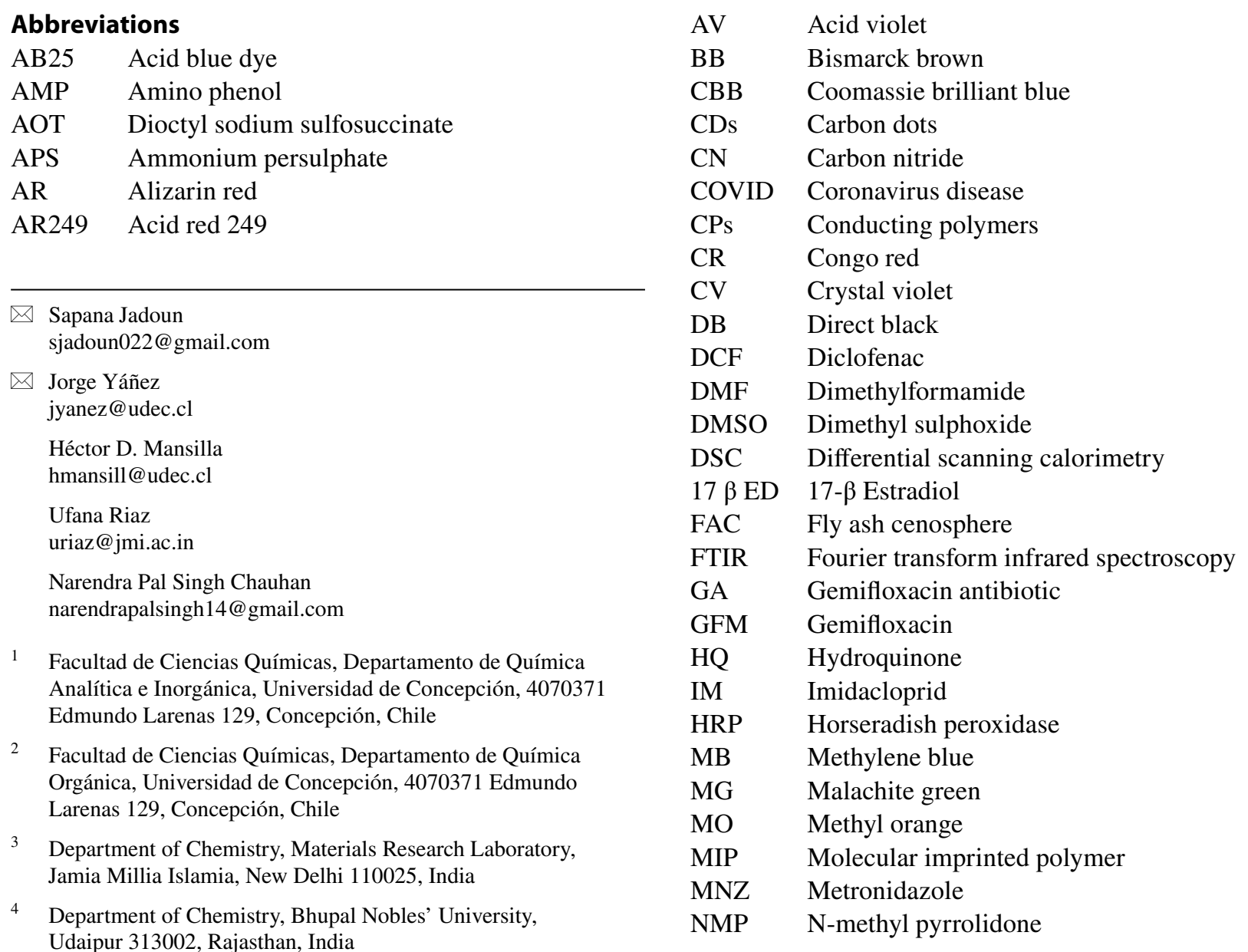




$\begin{array}{ll}\text { NMR } & \text { Nuclear magnetic resonance } \\ \text { OLEDs } & \text { Organic light-emitting diodes } \\ \text { OAA } & \text { Ortho-anthranilic acid } \\ \text { OAP } & \text { Ortho-aminophenol } \\ \text { OPD } & \text { Ortho-phenylenediamine } \\ \text { OTD } & \text { Ortho-toluidine } \\ \text { PAM } & \text { Poly(azomethine) } \\ \text { PAN } & \text { Polyacrylonitrile } \\ \text { PANI } & \text { Polyaniline } \\ \text { PAP } & \text { Para-aminophenol } \\ \text { PAZ } & \text { Poly(azomethyne) } \\ \text { PEDOT } & \text { Poly (ethylene dioxythiophene) } \\ \text { PEG } & \text { Poly (ethylene glycol) } \\ \text { PNA } & \text { Polynaphthylamine } \\ \text { POPD } & \text { Poly(ortho-phenylenediamine) } \\ \text { PPD } & \text { Poly(phenylenediamine) } \\ \text { PPPD } & \text { Poly (para-phenylenediamine) } \\ \text { PPY } & \text { Polypyrrole } \\ \text { PTH } & \text { Polythiophene } \\ \text { RB } & \text { Rhodamine B } \\ \text { R6G } & \text { Rhodamine 6-G } \\ \text { RO } & \text { Reactive orange } \\ \text { RR } & \text { Reactive red } \\ \text { SDS } & \text { Sodium dodecyl sulfate } \\ \text { SEM } & \text { Scanning electron microscope } \\ \text { TC } & \text { Tetracycline } \\ \text { TEM } & \text { Transmission electron microscopy } \\ \text { TGA } & \text { Thermogravimetric analysis } \\ \text { THF } & \text { Tetrahydrofuran } \\ \text { UV } & \text { Ultraviolet } \\ \text { WHO } & \text { World Health Organization } \\ \text { XRD } & \text { X-ray diffraction } \\ & \end{array}$

\section{Introduction}

"It isn't pollution that's harming the environment, it's the impurities in our air and water that are doing it", a well famous quote by Dan Quayle, Former US Vice President, best suits on today's situation where air and water get contaminated by several impurities due to anthropogenic activities. According to WHO (World Health Organization), the half population of the world will be forced to live in waterstressed areas by 2025. Contaminated water is directly associated with the cause of various disease transmissions such as cholera, hepatitis A, typhoid fever, diarrhea, lead poisoning, and COVID-19 (Organization 2020; Sharma et al. 2020; Ferreira et al. 2021; Huo et al. 2021; Lahrich et al. 2021). Nowadays, environmental remediation for polluted water due to organic pollutants, residual dyes from some industries and textiles, toxic metals incorporation in natural water resources is of great concern (Akpomie and Conradie 2020; Bhavya et al. 2021). Contaminated water has thoughtful consequences for living organisms as well as for the ecosystem; hence, an alarm has been raised for the scientific community to work for the removal of the organic contaminants before their discharge into the environment (Durgalakshmi et al. 2020; Hussain et al. 2021).

A lot of review articles are given for materials and methods which have been cast-off for the degradation and adsorption of those organic contaminants (Crini 2021; Kumar et al. 2021; Ponnuchamy et al. 2021; Vishnu et al. 2021). During the last few decades, heterogenous photocatalysis has been expanded rapidly for environmental remediation due to its simple design, stability, low set-up cost, and whole mineralization of pollutants into safe by-products (Luo et al. 2019; Madima et al. 2020). Water and air purification, solar water splitting are the most widely investigated fields in photocatalysis (Ke et al. 2018). Photocatalysis is used for the rapid and efficient destruction of environmental pollutants by using non-toxic semiconductors. This is also known as artificial photosynthesis in which sustainable chemistry plays a major role in resolving environmental and energy issues. This is a five-step process: (i) transfer of the reactant to the surface in the fluid phase, (ii) adsorption of reactant at the surface of the semiconductor, (iii) reactions in the adsorbed phase, (iv) decomposition or desorption of the products and finally (v) removal of the by-products at the interface region. The process is almost similar in conventional catalysis and heterogenous photocatalysis but the only difference is step (iii) in which mode of activation is changed to photonic activation in the case of heterogeneous photocatalysis and thermal activation in the case of conventional catalysis (Herrmann 1999; Kanamarlapudi et al. 2018).

For photocatalysis, inorganic semiconductors such as zinc oxide ( $\mathrm{ZnO}$ ) (Miranda et al. 2016; Acuña et al. 2017; Suresh et al. 2018), $\mathrm{TiO}_{2}$ (Nakata and Fujishima 2012; Pradenas et al. 2019), CdO (Amita et al. 2019), CdSe (Hiragond et al. 2018) and $\mathrm{MoS}_{2}$ (Li et al. 2018) showed immense potential and revealed noteworthy physicochemical and electronic properties applicable in photochromism and photovoltaics (Miranda et al. 2016). These inorganic semiconductors possess an empty conduction band and filled valance band in their ground state, when these are excited by more energy than their bandgap, the formation of excitons (holes in valence band and electrons in conduction band) occurs in the photocatalysis process (Talaiekhozani et al. 2020; Subudhi et al. 2021).

Among inorganic oxides, $\mathrm{ZnO}$ is one of the most extensively investigated photocatalytic semiconductors because of its unique features such as abundance, low toxicity, ecofriendly and cost-effectiveness (Yashni et al. 2020; Albiter et al. 2021). It has $60 \mathrm{meV}$ excitation binding energy and $3.37 \mathrm{eV}$ of direct wide bandgap at room temperature (Sharma 2020). Although in terms of eco-friendly and relative less toxicity, $\mathrm{ZnO}$ is a much better photocatalyst 
as compared to $\mathrm{TiO}_{2}, \mathrm{CdO}, \mathrm{CdSe}$, etc., however, on large scale, its practical applications are not that much appreciable because of reassimilation of photogenerated charge carriers at a high extent (Bera et al. 2019). These electron holes recombination favored the depleted quantum yield, and the absorption occurs only in the ultraviolet region due to the wide band gap of the semiconductor. The activation of $\mathrm{ZnO}$ nanoparticles occurs mainly in the ultraviolet region of the spectrum; hence, its use in sunlight irradiation is narrow. Therefore, shifting the absorption wavelength to the visible or near-infrared region is needed for improving its performance under solar irradiation. Also, ultraviolet radiations are harmful to the humans and environment (Bomila et al. 2019).

To overcome this problem, many efforts have been done in the field of modification of the surface of $\mathrm{ZnO}$ nanoparticles by numerous materials such as anionic and cationic doping, rare earth doping, co-doping, and couple semiconductor. In this regard, nanohybrids synthesized by conducting polymers and semiconductors have attracted a lot of attention from researchers (Sandoval et al. 2019). To achieve good charge separation efficiency along with good charge transfer, the nanohybrids of $\mathrm{ZnO}$ with conducting polymers such as polyaniline (Mozafari and Chauhan 2019; Deng et al. 2020), polyphenylendiamine (Khokhar et al. 2020a, 2021), polypyrrole (Jadoun et al. 2018), poly(1-naphthylamine) (Jadoun et al. 2017b, c) having unique thermal, optical, electrical, mechanical, biomedical and catalytic properties which have been extensively employed in photocatalysis for degradation of organic contaminants, dyes, and reduction of toxic metals. Here, conducting polymers act as a sensitizer for $\mathrm{ZnO}$ nanoparticles. Energy levels of conducting polymers match with the $\mathrm{ZnO}$ and both can be excited by visible light to produce photogenerated carriers, Fig. 1 (Ghosh 2021). Consequently, they can absorb appropriately sunlight for solar photocatalysis to improve sustainability. Therefore, it is preferable and economical with the use of nanohybrid photocatalyst of conducting polymers and $\mathrm{ZnO}$ for rapid and efficient destruction of environmental pollutants (Riaz et al. 2015).

Here, we review the various nanohybrids comprising conducting polymers and $\mathrm{ZnO}$ which are specially synthesized for efficient photocatalysis to overcome the photocorrosion tendency of $\mathrm{ZnO}$ and inactivation under ultraviolet light illumination occurred in hollow $\mathrm{ZnO}$ core/ZnS shell structure (Yu et al. 2015), while this drawback was overcome by using polyaniline in form of nanohybrids of polyaniline/ZnS/ZnO in which no reduction of photocurrent was achieved and photo-corrosion was inhibited by using polymer (Kim et al. 2020).

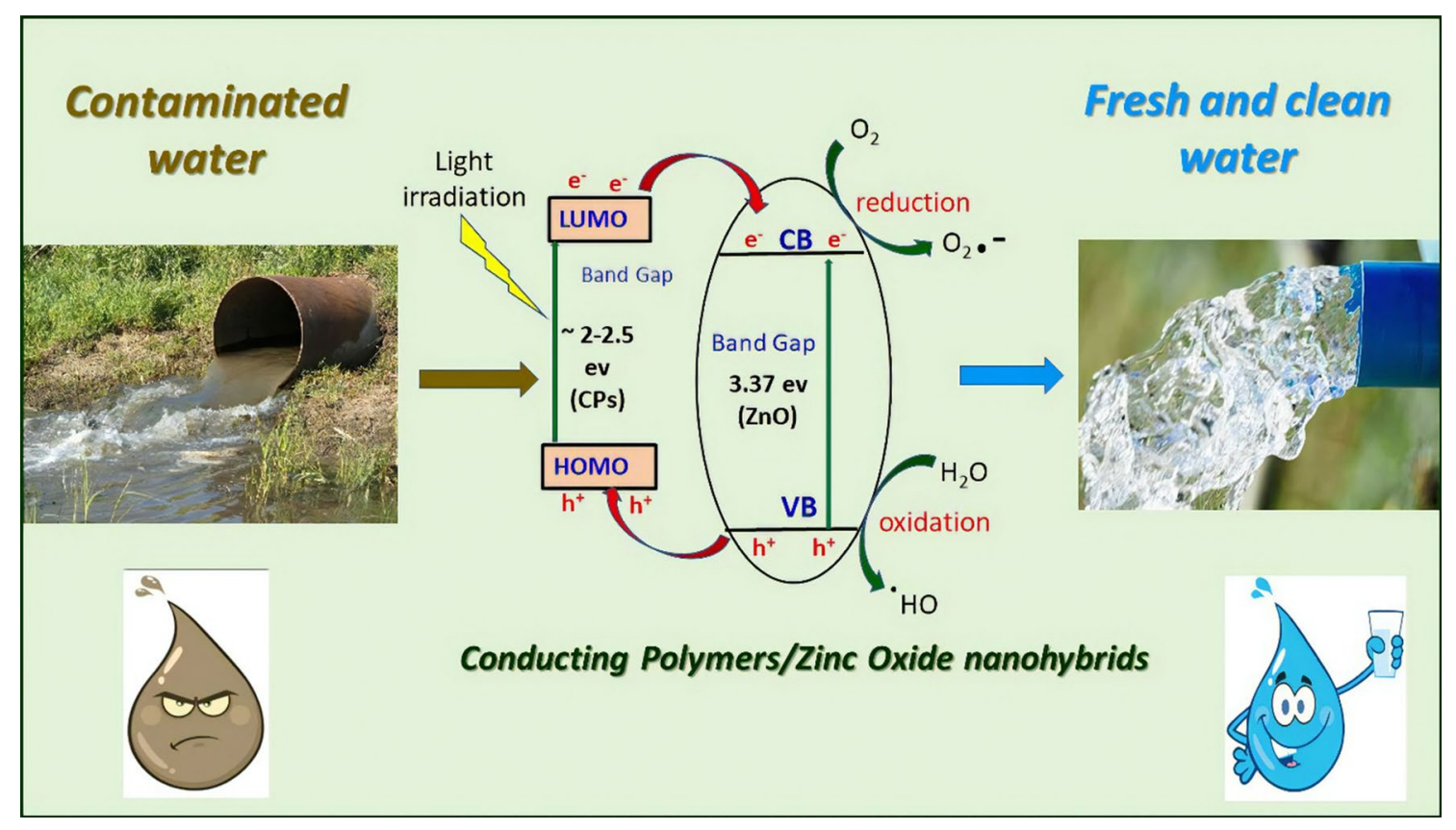

Fig. 1 Conducting polymers/zinc oxide-based photocatalysis for environmental remediation including the bandgap and transfer of electrons for complete photocatalysis by nanohybrids. Here, the conducting polymers possess low band gap of $2-2.5 \mathrm{eV}$, while $\mathrm{ZnO}$ has wide band gap and shows synergistic relation to each other for transfer of electrons. CB: conduction band; VB: valence band; HOMO: highest occupied molecular orbital; LUMO: Lowest unoccupied molecular orbital; CPs: conducting polymers; $\mathrm{ZnO}$ : zinc oxide 


\section{Photocatalysis}

The combination of photochemistry and catalysis is known as photocatalysis. Photocatalysis involves the light and catalyst simultaneously to boost any chemical reaction (Iervolino et al. 2020; Rueda-Marquez et al. 2020). The phenomena of photocatalysis were firstly discovered by Fujishima and Honda in 1972 on $\mathrm{TiO}_{2}$ surface by water splitting under ultraviolet light and referred to as Honda-Fujishima Effect (Fujishima and Honda 1972; Hashimoto et al. 2005). However, electrochemists initially discarded this event due to their thought about the generation of oxygen is not possible at low voltage. They mentioned that electrolysis of water occurs at a minimum of 1.5-2 V. Afterthought, wide research was carried out by researchers in various fields and came to know that these studies were associated with renewal and storage of energy. With his discovery of such a photocatalyst, Prof. Fujishima said for the society "The primary objective of science and technology is to create a society where people can have healthy, comfortable, and long lives. The crucial thing in science and technology is to develop a new concept that can be applied to actual products and services, and these new products and services will eventually make people happy" (Fujishima et al. 2000). From that time photocatalysis was rapidly grown for the degradation of contaminants in wastewater (Fujishima et al. 2008).

Generally, photocatalysis is a light-induced redox reaction in which the surface of the catalyst is exposed to the light source to excite the valence band electrons and transferred to the conduction band, leaving a hole in the valence band. The hole in the valence band can oxidize the water molecules by creating hydroxyl radicals and excited electrons in the conduction band can reduce the absorbed oxygen on the surface of the photocatalyst. Additionally, the photogenerated holes can directly oxidize organic matter (Palominos et al. 2008).

The redox process occurred at the surface of the catalyst. The absorbed water remains on the photocatalyst surface, and it is oxidized by the holes (present in the valence band after excitation of the electron to conduction band) resulting in the hydroxyl radicals $(\mathrm{OH})$. The hydroxyl radicals further come in contact for reaction with organic contaminates, and after radical chain reactions, the organic contaminants degrade in carbon dioxide and water. This whole process is known as the oxidation process. In the reduction process, the oxygen reacts with electrons present in the conduction band to produce superoxide anions which further attach to the intermediate products of oxidative reaction to produce peroxide or convert to hydrogen peroxide to water. In organic matter, the reduction is much easier than water, and hence, the number of positive holes increased with the increment of concentration of organic matter which decreases the recombination of charges and increases the photocatalysis (Saravanan et al. 2017).

To overcome the recombination of charge carriers during photocatalysis, semiconductors are combined with conducting polymers possessing a low bandgap and absorb light in both ultraviolet and visible regions. The nanohybrids possessed two distinct energy levels to play a key role in charge separation. In nanohybrids of conducting polymers and $\mathrm{ZnO}$, conducting polymers absorb a wide range of visible light and act as a photosensitizer due to having a lower bandgap of conducting polymers compared to the $\mathrm{ZnO}$. The electrons which reached from HOMO (highest occupied molecular orbital) to LUMO (lowest unoccupied molecular orbital) of conducting polymers by excitation are finally transferred to conduction band of $\mathrm{ZnO}$ and react with the molecular oxygen to produce $\mathrm{O}_{2}^{--}$, while the holes in HOMO (highest occupied molecular orbital) of conducting polymers react with water to produce ' $\mathrm{OH}$ to increase the efficiency of photocatalysis. Therefore, photocatalysis using the nanohybrids with various combinations of conducting polymers and $\mathrm{ZnO}$ seems to be the best alternative to remove the water pollutants/dyes by inhibiting the possibility of recombination of charge carriers and enhancing the potential for redox reaction at the surface of photocatalyst.

\section{Synthesis of conducting polymer/zinc oxides composites}

There are various methods to synthesize conducting polymers/ZnO nanohybrids for active photocatalysis which are as follows.

\section{In situ polymerization}

In situ polymerization is one of the best and successful techniques for the preparation of nanohybrids, to attain great interfacial interactions between conducting polymers and $\mathrm{ZnO}$ and it means "in the polymerization mixture." In this method, polymer nanohybrids are achieved via the mixing of semiconductor in a solution containing monomer followed by polymerization, and at the same time, semiconductor and matrix make the covalent linkage (Abinaya et al. 2019; Mittal et al. 2019). In situ polymerization starts with the initiation step followed by many polymerization steps resulting in the formation of nanohybrids. The addition of an initiator or oxidizing/reducing agent starts the polymerization reaction by this homogenous mixture when exposed to any heat, radiation, etc., followed by washing and drying of nanohybrids. On completion of the polymerization reaction, a polymer nanocomposite is fashioned consisting of the 
bonding between polymer and nanoparticle (Abinaya et al. 2019; Mittal et al. 2019).

Several research articles included in situ polymerization method for synthesis of conducting polymers/ $\mathrm{ZnO}$ as it is an easy method without any hurdles (Demir et al. 2006; Ameen et al. 2011; Ramesan et al. 2017; Ponnamma et al. 2019). Gilja and coworkers (Gilja et al. 2018) synthesized polyaniline/ZnO nanocomposites in various ratios by using the same method at room temperature with water and diethylene glycol mixture at neutral $\mathrm{pH}$. $\mathrm{ZnO}$ nanoparticles were taken in solution, and aniline monomer was added in the same mixture followed by oxidizing agent ammonium persulphate. The concentration of diethylene glycol was taken $0.02,0.024$, and $0.04 \mathrm{M}$, while the molar ratio of aniline (monomer) and oxidant was taken 1:0.25, aniline, and $\mathrm{ZnO}$ was taken in a weight ratio of 15:85. Initially, a fixed amount of aniline, $\mathrm{ZnO}$, and diethylene glycol was taken in a flask containing $25 \mathrm{ml}$ of distilled water and allowed to react for $15 \mathrm{~min}$ at ultrasonicator to achieve stable suspension followed by the addition of ammonium persulphate to start polymerization, and the reaction was carried out for $2 \mathrm{~h}$. Finally, these were washed several times by water and centrifuged then dried at $60^{\circ} \mathrm{C}$.

Olad et al. (OLAD et al. 2012) also used the in situ oxidative polymerization method to synthesize polyaniline/ $\mathrm{ZnO}$ nanohybrids with the dispersion of $0.9313 \mathrm{~g}$ of $\mathrm{ZnO}$ nanoparticles in the presence of $0.01 \mathrm{~mol}$ of aniline monomer and $0.01 \mathrm{~mol}$ of hydrochloric acid with ammonium persulphate $(0.01 \mathrm{~mol})$ as an oxidant. The nanohybrids were able to degrade $82 \%$ of methylene blue dye in $60 \mathrm{~min}$. The nanohybrids of poly(p-phenylenediamine) with $\mathrm{ZnO}$ were synthesized by Rostami and labmates by using ammonium persulphate as an oxidant. (Rostami et al. 2015). Some other researchers also adopted the same method for the synthesis of conducting polymers/ $\mathrm{ZnO}$ nanohybrids (Eskizeybek et al. 2012; Haldorai et al. 2014; Sivakumar et al. 2014).

In situ polymerization method has many advantages such as cost-effective materials used in the reaction, easy to handle, and being compatible during various curing and heating methods. Also, strong covalent interaction between polymers and semiconductors occurs using this method.

\section{Sol-gel synthesis}

The sol-gel synthesis method can be adopted to change or to improve the properties of nanoparticles (Faisal et al. 2020). For the synthesis of conducting polymers/ $\mathrm{ZnO}$ nanohybrids via sol-gel preparation method, zinc nitrate was mixed with citric acid to make a solution and stirred for $3 \mathrm{~h}$ at $80{ }^{\circ} \mathrm{C}$ and metal citrate was formed. The ammonia solution ( $\mathrm{pH} 4.5)$ was added to the above reaction mixture and stirred continuously for $6 \mathrm{~h}$. After completion of the reaction, a complex of citrate of zinc was formed in the spongy gel form which was washed several times with acetone and distilled water, further heated for $1 \mathrm{~h}$ at $150{ }^{\circ} \mathrm{C}$. The obtained material was ground and calcinated to remove the impurities. After that, the synthesis of polyaniline/ $\mathrm{ZnO}$ was done by the oxidative polymerization method in which aniline monomer $(0.1 \mathrm{M})$ was mixed with hydrochloric acid (1 M) to produce aniline hydrochloride. In this step, $\mathrm{ZnO}$ was added in a fixed amount and $0.1 \mathrm{M}$ ammonium persulphate was added dropwise for polymerization and the temperature of the reaction was maintained at $4{ }^{\circ} \mathrm{C}$ using ice. The synthesized sample was washed several times and dried in a vacuum oven for $24 \mathrm{~h}$ (Ambalagi et al. 2016). The whole process is shown in form of a flowchart in Fig. 2.

Likewise, Patil and their lab mates adopted the sol-gel synthesis method. Firstly, they prepared polyaniline by using the chemical oxidative polymerization method with $2 \mathrm{ml}$ of aniline monomer in $1.0 \mathrm{M}$ hydrochloric acid. $5.393 \mathrm{~g}$ of zinc acetate was used in $40 \mathrm{ml}$ of methanol and stirred continuously at $60{ }^{\circ} \mathrm{C}$ for the synthesis of $\mathrm{ZnO}$ nanoparticles in gel form. The heating was stopped after $1 \mathrm{~h}$ and the reaction was continuous until the white powder was formed. Further, the polyaniline powder previously synthesized was dissolved in $m$-cresol and stirred followed by the filtration of the solution with a few microns size Whatman filter paper. After that, the film of polyaniline was deposited on a glass substrate by spin coating method for $30 \mathrm{~s}$ at $2000 \mathrm{rpm}$ and dried at $100{ }^{\circ} \mathrm{C}$ on a hot plate, while the filtered solution of polyaniline was allowed to react with various percent solutions of $\mathrm{ZnO}$ on stirrer for $11 \mathrm{~h}$ to form nanohybrids of polyaniline $/ \mathrm{ZnO}$ (Patil et al. 2012). The sol-gel method for the synthesis of nanohybrids is simpler and cheaper than others but much time is needed to optimize the stability and durability of sol which is needed to be overcome.

\section{Template synthesis}

Numerous nanohybrids of conducting polymers/ZnO were fabricated by using some templates. To get the nanohybrids some post-synthetic procedures are needed to eliminate the template used in synthesis. In this regard, firstly, $\mathrm{ZnO}$ nanoparticles were synthesized using sodium dodecyl sulfate as a template via precipitation method in a bicontinuous microemulsion system. $40.5 \mathrm{wt} \%$ of surfactant and $32.5 \mathrm{wt} \%$ of $0.7 \mathrm{M}$ zinc nitrate solution were allowed to react in toluene and water mixture on magnetic stirrer followed by addition of aqueous $\mathrm{NaOH}$ solution by dosing pump in $15 \mathrm{~min}$. After 30 min of reaction, acetone was added to get precipitate of $\mathrm{ZnO}$ nanoparticles which was washed and dried. $250 \mathrm{mg}$ of $\mathrm{ZnO}$ nanoparticles was taken in an aqueous solution having $0.8 \mathrm{~g}$ of sodium dodecyl sulfate followed by the addition of $0.4 \mathrm{~g}$ of pyrrole and allowed to react for $2 \mathrm{~h}$.

To start the polymerization, ammonium persulphate $(0.6 \mathrm{M})$ was added to the reaction mixture. Methanol was 


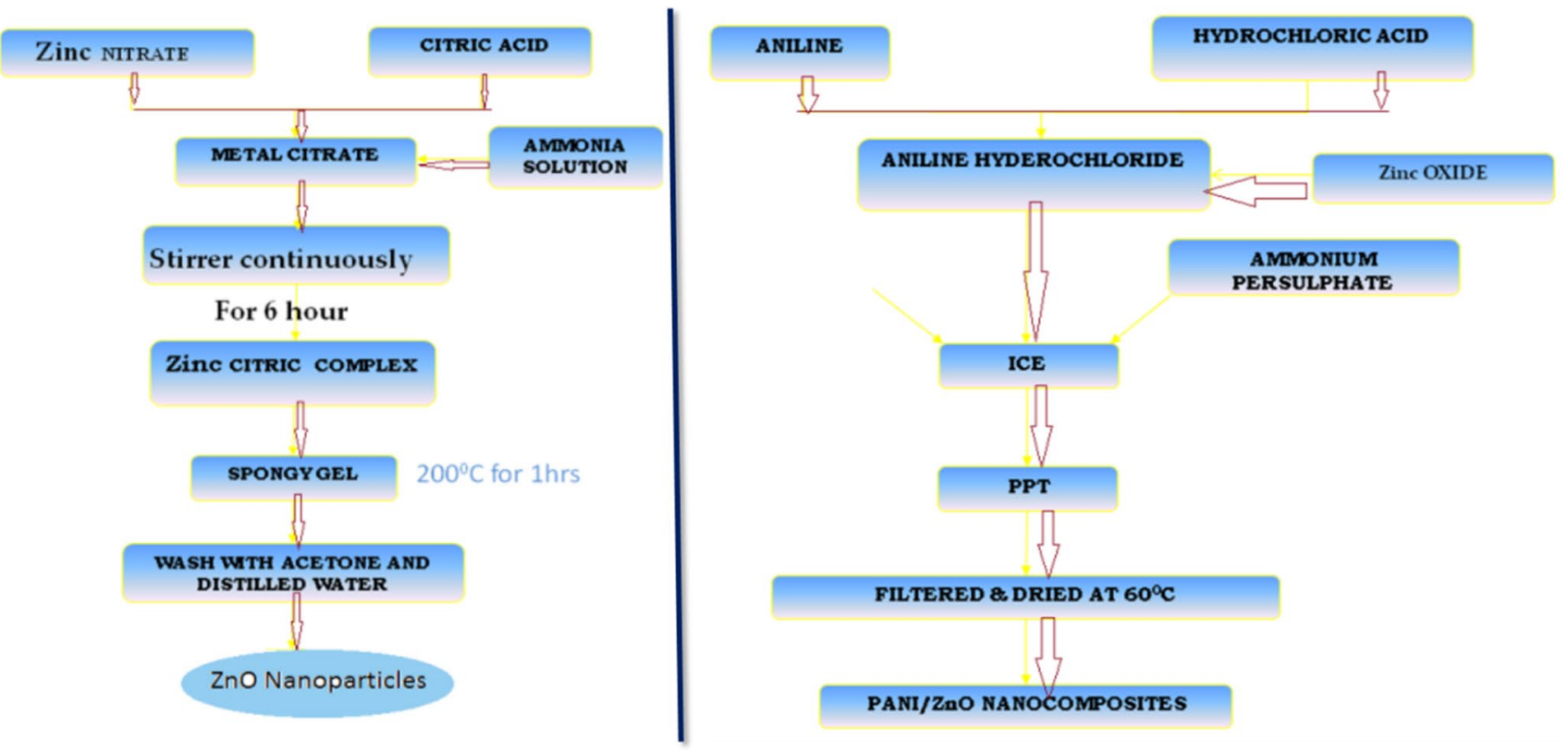

Fig. 2 Synthesis of zinc oxide nanoparticles using zinc nitrate and citric acid followed by the synthesis of polyaniline/zinc oxide nanohybrids by sol-gel method by using aniline monomer and zinc oxide

in hydrochloric acid medium (Reprinted from Ambalagi et al. 2016 with permission from Elsevier). PANI: polyaniline; $\mathrm{ZnO}$ : zinc oxide

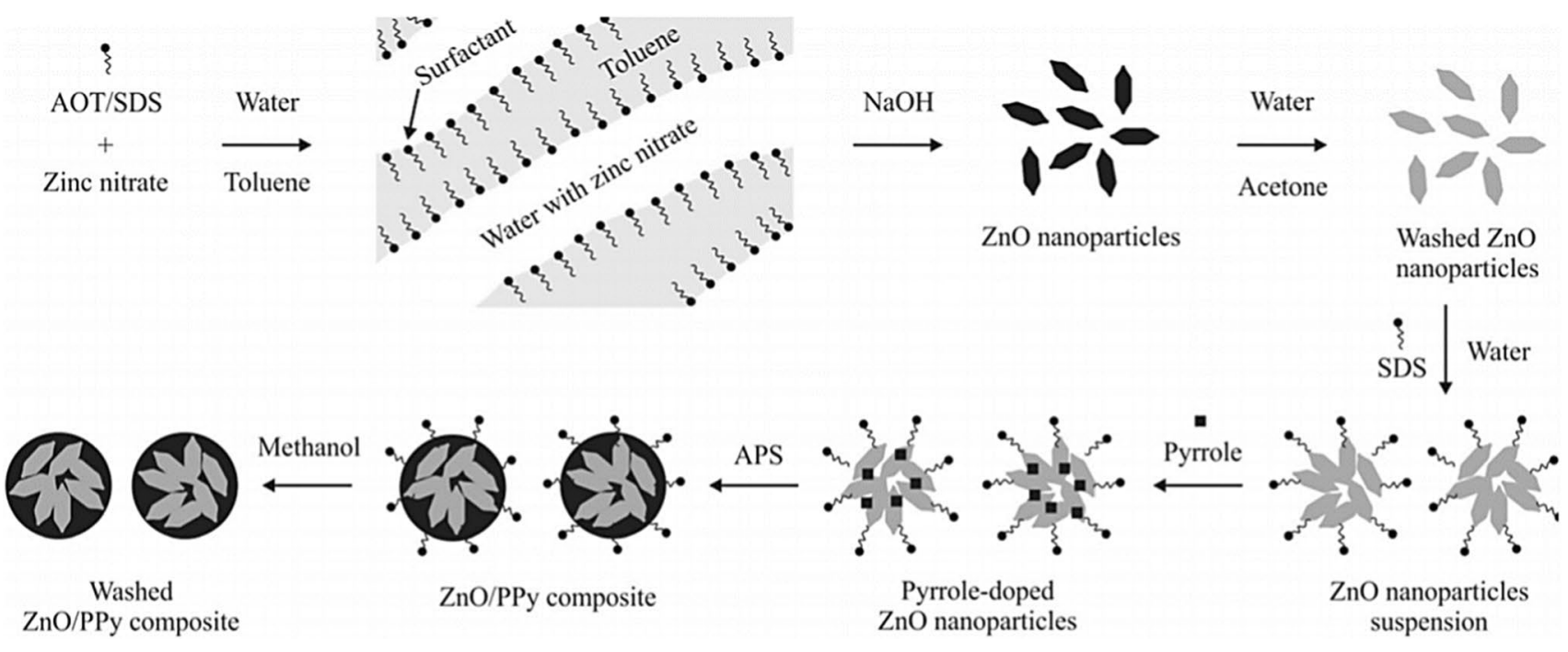

Fig. 3 Template synthesis of polypyrrole /zinc oxide nanohybrids using sodium dodecyl sulfate as template and ammonium persulphate as oxidant. Here, synthesis of zinc oxide nanoparticles was performed using zinc nitrate in the AOT/SDS template followed by nanohybrids

used to separate the nanohybrids after $1 \mathrm{~h}$ of reaction, as shown in Fig. 3 (Ovando-Medina et al. 2015). Zhao and coworkers also synthesized polyaniline/ZnO nanohybrids via this method using the combination of wet-chemical technique in situ polymerization route (Zhao et al. 2011). Some other researchers have also adopted this route for synthesis. Reprinted from Ovando-Medina et al. 2015 with permission from Springer). AOT: dioctyl sodium sulfosuccinate; $\mathrm{NaOH}$ : sodium hydroxide; ZnO: zinc oxide; PPy: polypyrrole; APS: ammonium persulphate; SDS: sodium dodecyl sulfate

conducting polymers / $\mathrm{ZnO}$ nanohybrids synthesis (Mitra et al. 2017; Zoshki et al. 2019). Template synthesis is an efficient method for controlling the morphology and size but the only difficulty is to remove the template after synthesis which limits its use and needs to be worked on that in the future. 


\section{Mixing or blending of polymer and nanoparticles}

Direct mixing of polymer and nanoparticles is another method for the fabrication of nanohybrids. This method includes the direct mixing of polymer with $\mathrm{ZnO}$ nanoparticles. Firstly, the synthesis of polymer and $\mathrm{ZnO}$ occurs separately, afterthought the mixing of both can be done via various techniques. For this, polyaniline was synthesized by its monomer using $0.5 \mathrm{M}$ of hydrochloric acid and $0.05 \mathrm{M}$ of ammonium persulphate. The reaction was carried out for $6 \mathrm{~h}$, and the resultant precipitate was washed and dried at $70{ }^{\circ} \mathrm{C}$ for $30 \mathrm{~h}$. The obtained polyaniline was in emeraldine salt form (doped) and was washed with ammonia solution to achieve polyaniline in emeraldine base form. For the synthesis of $\mathrm{ZnO}$ rods, the hydrothermal method was chosen. In this method, $0.2 \mathrm{M}$ zinc sulphate $(25 \mathrm{ml})$ solution and $4 \mathrm{~g}$ of sodium hydroxide $(25 \mathrm{ml})$ were mixed with deionized water $(50 \mathrm{ml})$. This mixture was stirred for $30 \mathrm{~min}$ and kept on stainless steel autoclave followed by keeping in an oven for $10 \mathrm{~h}$ at $80^{\circ} \mathrm{C}$. Later on, this precipitate was washed with ethanol and water several times and dried in an oven for $12 \mathrm{~h}$ at $80^{\circ} \mathrm{C}$. In the last step, $0.05 \mathrm{~g}$ of polyaniline was taken in $100 \mathrm{ml}$ of THF (tetrahydrofuran), and the solution containing polyaniline was ultrasonicated for $30 \mathrm{~min}$ and stirred again. At this stage, $\mathrm{ZnO}$ was added to the mixture followed by stirring of mixture for $24 \mathrm{~h}$. After completion of the reaction, precipitates were washed with deionized water and kept for drying at $60{ }^{\circ} \mathrm{C}$ (Sharma et al. 2016).

The blending of polymers and semiconductors is the most effortless way for the nanohybrid synthesis as only mechanical mixing of polymer and semiconductor is required in this technique, but the main disadvantage of this method is the lack of bonding and interactions between polymer and semiconductor compared to other above methods. To overcome this problem, some such techniques should be designed that can deeply blend for interaction between polymer and semiconductors or nanomaterials.

\section{Photocatalysis by conducting polymer/zinc oxide nanohybrids}

Conducting polymers are the class of conjugated polymers having the $\pi$-conjugated system in their backbone and have a wide area of applications in the field of optoelectronics (Khokhar et al. 2020b), biomedical (Jadoun et al. 2021c), photovoltaic devices (Jadoun and Riaz 2020), etc., due to their unique optical, electrical, and photoelectric properties (Jadoun et al. 2021b). Conducting polymers are extensively used as a photosensitizer to enhance the semiconductor's photocatalytic properties (Jangid et al. 2020b). Some conducting polymers combined with $\mathrm{ZnO}$ to form nanohybrids for the photocatalytic degradation of pollutants from water are discussed here and summarized in Table 1 and Fig. 4.

\section{Polyaniline/zinc oxide}

Polyaniline is a mostly studied conducting polymer among all due to its outstanding properties and multiple applications (Chauhan et al. 2016; Jangid et al. 2020a). It is used widely used to tune the electrical, optical, and photocatalytic properties of composites with $\mathrm{ZnO}$ due to its narrow bandgap of $2.13 \mathrm{eV}$. The polyaniline/ $\mathrm{ZnO}$ nanohybrids are the extensively explored class of all conducting polymers/ $\mathrm{ZnO}$. These were synthesized in various media and neutral is one of them studied by some researchers. They concluded that the synthesis in neutral media with DEG enhanced the solubility of aniline in water and forms dispersion which was more stable and have diminished bandgap. These photocatalysts were able to react under solar irradiation.

During photocatalysis, the corrosion dissolution of $\mathrm{ZnO}$ was also prevented by this method. These photocatalysts had shown high catalytic activity and stability for the removal of the acid blue dye from model wastewater. Upon light irradiation, electrons got excited from HOMO of polyaniline and transferred to LUMO and further easily transferred to the conduction band of $\mathrm{ZnO}$ due to well-matched energy levels of both. Hence, holes reacted with water and electrons reacted with oxygen to form reactive oxygen species such as $\mathrm{OH}, \mathrm{O}_{2}^{-\cdot}, \mathrm{HO}_{2}{ }^{-}$, and the most active radical was $\mathrm{OH}$ which was produced by the reaction between hydroxyl ions and holes. These were able to degrade organic pollutants and finally produce $\mathrm{CO}_{2}$ and $\mathrm{H}_{2} \mathrm{O}$, Fig. 5 (Gilja et al. 2018).

The photocatalytic activity was found to enhance with the polyaniline and $\mathrm{ZnO}$ combination due to the synergic effect between polyaniline and $\mathrm{ZnO}$. These nanohybrids were 2.5 times more photocatalytically active as compared to $\mathrm{ZnO}$ for the degradation of methyl orange dye under ultraviolet light (Pei et al. 2014). These nanohybrids were also capable to remove toxic and stable pollutants such as 4-chlorophenol. On ultraviolet irradiation, it removed organic carbon species up to $82 \%$ in $120 \mathrm{~min}$. In natural sunlight, these nanohybrids degraded $97 \%$ and $99 \%$ of methylene blue and malachite green, respectively, in $5 \mathrm{~h}$ (Eskizeybek et al. 2012). Zhu and coworkers fabricated polyacrylonitrile/polyaniline nanofibers and later $\mathrm{ZnO}$ was immobilized by atomic layer deposition to enhance the photocatalytic degradation of MB under UV light. The photocatalyst was easily recycled with very less decrement of photocatalytic activity. (Zhu et al. 2018).

To resolve existing energy or environmental hitches, architecture and structural design also played a significant role in a nanohybrid photocatalyst to enhance photocatalytic efficiency. In this regard, a novel polyaniline $/ \mathrm{ZnO}-\mathrm{CoMoO}_{4}$ $\mathrm{Z}$-scheme photocatalyst was fabricated and applied to the degradation of imidacloprid pesticide under visible light 


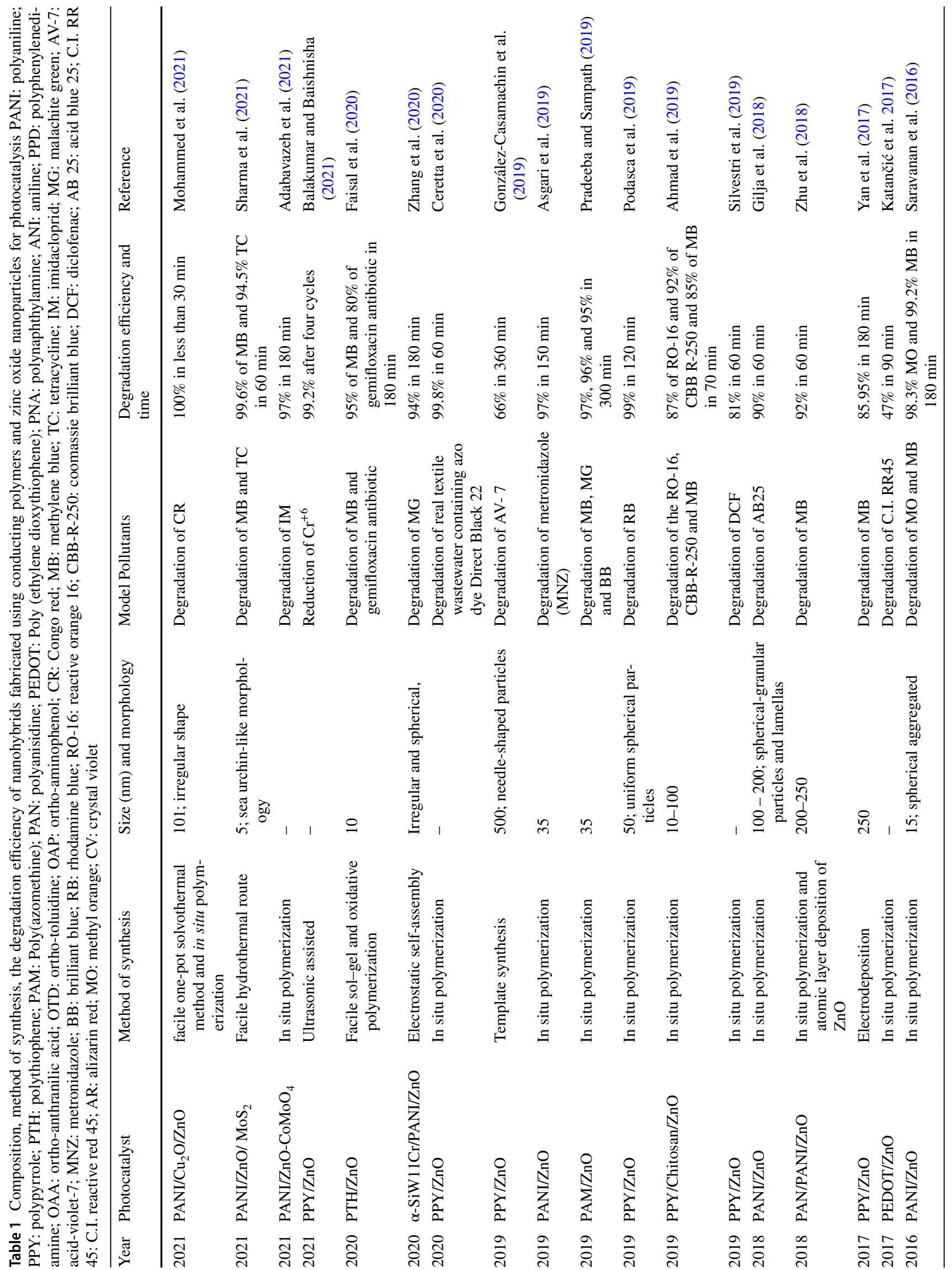




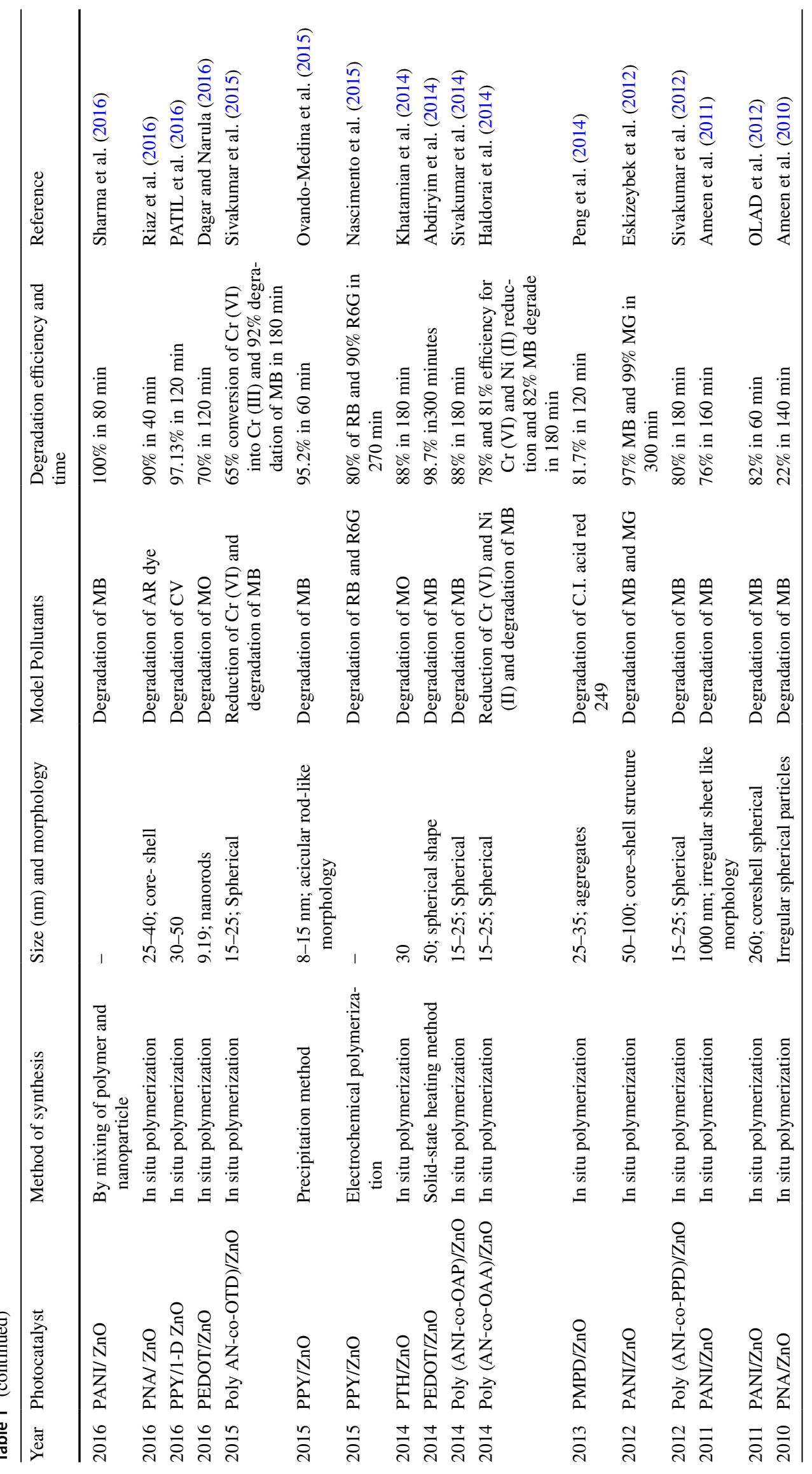


Fig. 4 Conducting polymers/ zinc oxide nanohybrids, their synthesis methods such as in situ polymerization, template synthesis, sol-gel synthesis and mixing or blending of polymer with nanoparticles, and model pollutant. PANI: polyaniline; PNA: polynaphthylamine; PPY: polypyrrole; ANI: aniline; PPD: polyphenylenediamine; OAA: ortho-anthranilic acid; AN: Anisidine; OTD: ortho-toluidine; OAP: ortho-aminophenol; PAM: Poly(azomethine); PTH: polythiophene; PEDOT: Poly (ethylene dioxythiophene); MB: Methylene blue; MG: malachite green; BB: brilliant blue; AR: alizarin red; MNZ: metronidazole; $\mathrm{AB}$ 25: acid blue 25; MO: methyl orange; CR: Congo red; TC: tetracycline; IM: imidacloprid; RO-16: reactive orange 16; CBB-R-250: coomassie brilliant blue; AV-7: acid-violet-7; DB-22: direct black-22; RB: rhodamine blue; DCF: diclofenac; R6G: rhodamine 6 G; C.I. AR 249: C.I acid blue 249; C.I. RR 45: C.I. reactive red 45 ; $17 \beta$-ED: $17-\beta$ estradiol; GA: Gemifloxacin antibiotic

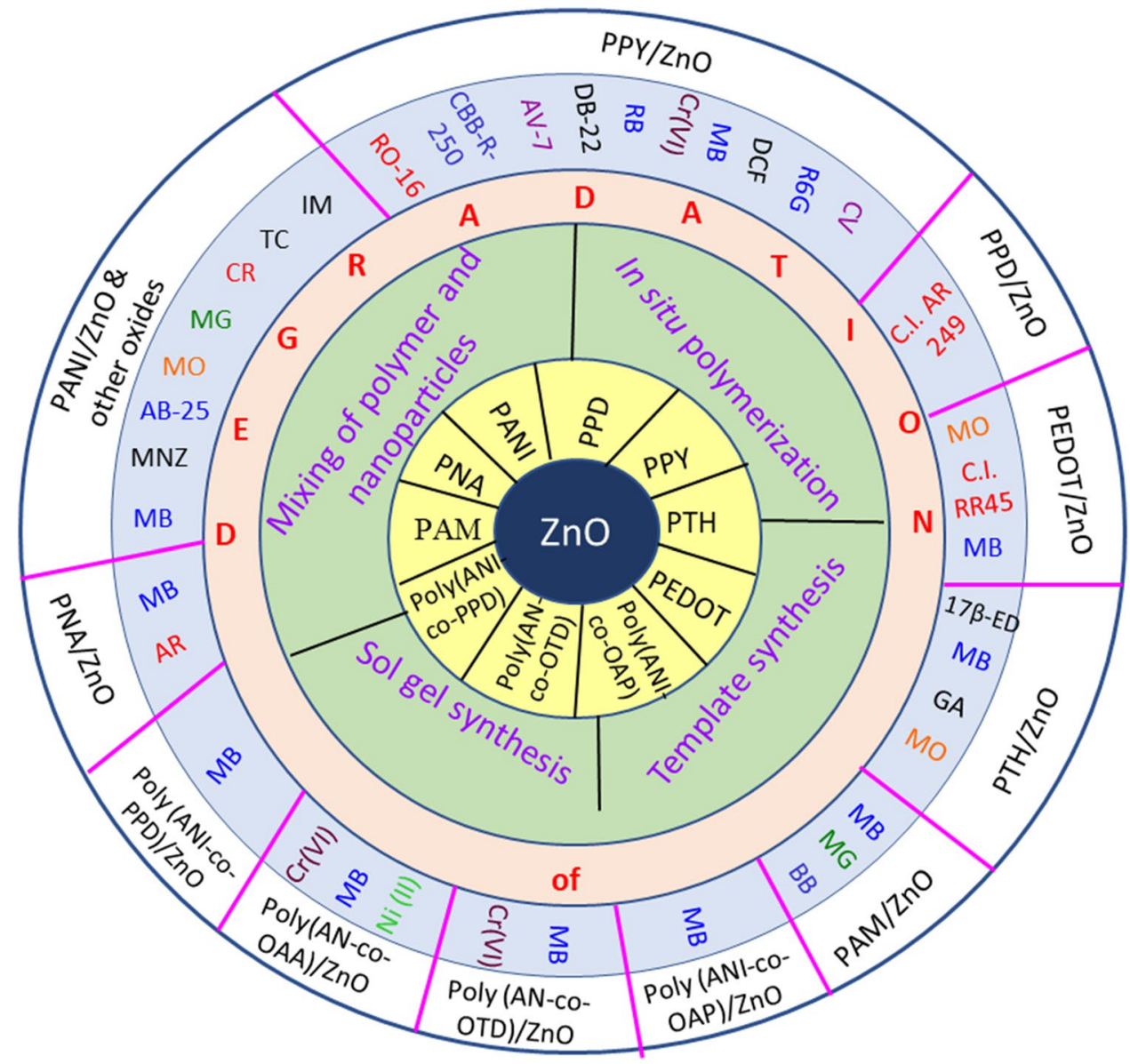

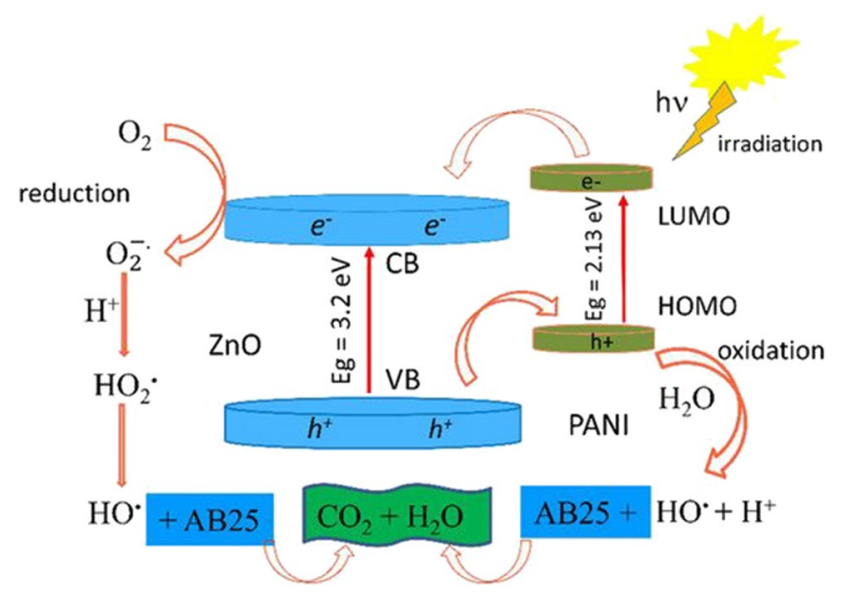

Fig. 5 Photocatalytic degradation mechanism of AB25 using polyaniline /zinc oxide composites. Here, polyaniline acts as a sensitizer and the transfer of electrons from LUMO of polyaniline to conduction band of zinc oxide, while holes are transferred from valence band of zinc oxide to HOMO diminished the electrons and holes recombination. Reprinted from Gilja et al. 2018 with permission from MDPI). CB: conduction band; VB: valence band; PANI: polyaniline; HOMO: highest occupied molecular orbital; LUMO: lowest unoccupied molecular orbital; AB25: acid blue dye 25 showing outstanding results. The predicted and experimental degradation was achieved up to 97.1 and $97.38 \%$ within $180 \mathrm{~min}$, and the photocatalyst was easily recovered and reusable (Adabavazeh et al. 2021).

Photocatalytic properties, stability, reusability, and adsorption properties were enhanced by $\mathrm{Cu}_{2} \mathrm{O} / \mathrm{ZnO} /$ polyaniline, a Z-scheme heterojunction properties ternary composite. The surface area of composites was increased up to $45.32 \mathrm{~m}^{2} \mathrm{~g}^{-1}$ which was high in comparison to pristine $\mathrm{Cu}_{2} \mathrm{O}, \mathrm{ZnO}$, and polyaniline. These composites revealed excellent $100 \%$ degradation of Congo red dye in less than 30 min which was a milestone in photocatalysis and even for reusability these were still active after the fifth cycle with $92 \%$ efficiency. In $\mathrm{Z}$ scheme heterojunction, there is always a semiconductor with electron donor and acceptor moieties, and hence, upon light irradiation, the electrons and holes are transferred from one semiconductor to another semiconductor via electron donor and acceptor moieties. As result, generation of higher redox ability due to electrons and holes on the semiconductor occurs by which charge separation of carriers occurred effectively and caused retention of sturdy oxidation-reduction potential for a long time.

Due to this architecture of $\mathrm{Z}$ scheme heterojunction, the surface area of composite increased as well as quenching 
(a)

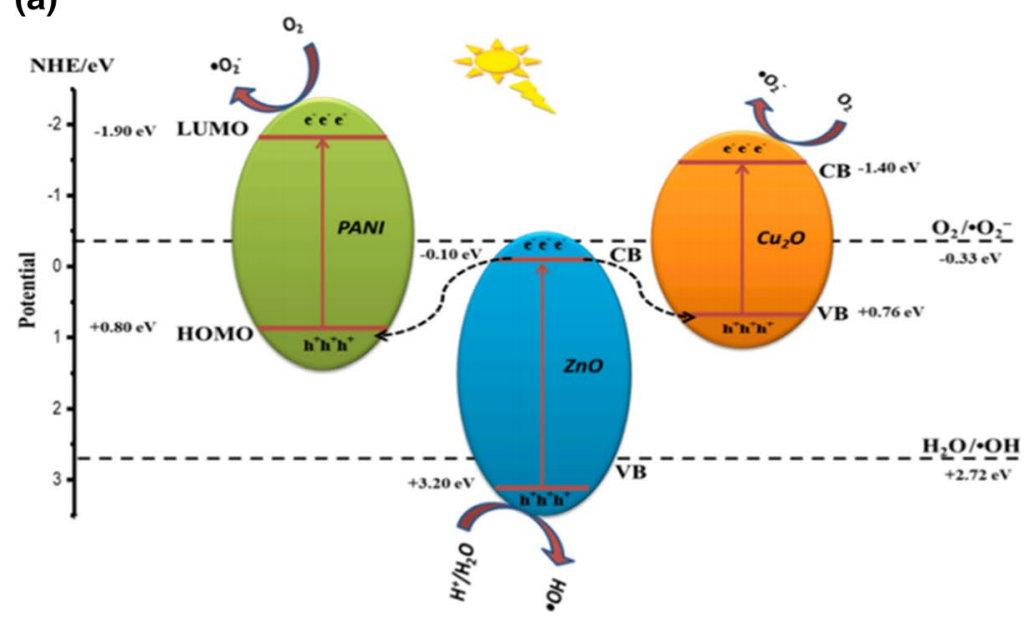

(b)

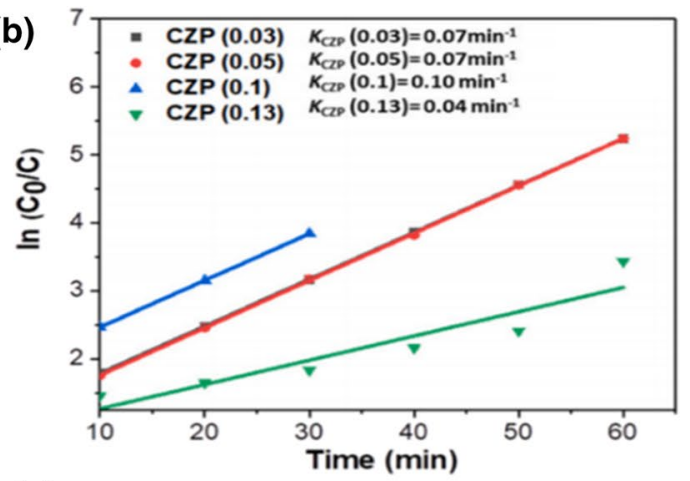

(c)

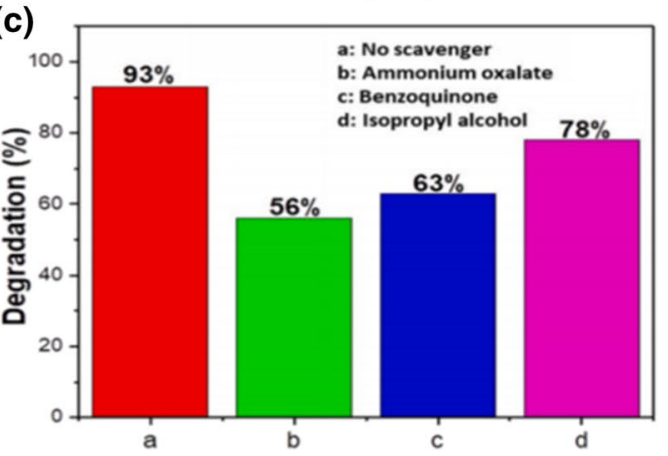

Fig. 6 Charge transfer pathway of a double $\mathrm{Z}$-scheme $\mathrm{Cu}_{2} \mathrm{O} / \mathrm{ZnO}$ / polyaniline ternary composite $(\mathrm{CZP})$ for oxidation and reduction under visible-light irradiation. $\mathbf{b}$ the rate constant of photocatalytic activity on Congo red dye by $\mathrm{Cu}_{2} \mathrm{O} / \mathrm{ZnO} /$ polyaniline ternary composite (CZP) at different loadings, $\mathbf{c}$ effects of scavengers on the degradation of Congo red on to $\mathrm{Cu}_{2} \mathrm{O} / \mathrm{ZnO} /$ polyaniline ternary compos-

of photoluminescence also favored the decrease in recombination of charge carriers, Fig. 6a. For the comparison, $\mathrm{Z}$ scheme $\mathrm{Cu}_{2} \mathrm{O} / \mathrm{ZnO} /$ polyaniline ternary composite was prepared in the various ratios of polyaniline, and the best results were shown by $\mathrm{Cu}_{2} \mathrm{O} / \mathrm{ZnO} /$ polyaniline ternary composite (0.1) in terms of maximum rate constant $\left(0.10 \mathrm{~min}^{-1}\right)$ and the least half-life (9.93 min), Fig. 6 (b), supported the highest photocatalytic activity of $\mathrm{Cu}_{2} \mathrm{O} / \mathrm{ZnO} /$ polyaniline ternary composite (0.1). The active species involved in the degradation of CR such as hydroxyl radicals $(\mathrm{OH})$, holes $\left(\mathrm{h}^{+}\right)$ and superoxide radicals $\left(\mathrm{O}^{--}\right)$were captured by isopropyl alcohol $(2 \mathrm{~mL})$, benzoquinone $(0.001 \mathrm{M})$, and ammonium oxalate $(0.001 \mathrm{M})$ in radical scavenging experiment and revealed the degradation in order of no scavenger $>$ isopropyl alcohol $>$ benzoquinone $>$ ammonium oxalate, Fig. $6 c$. The results showed that superoxide radicals $\left(\mathrm{O}_{2}^{-\cdot}\right)$ and holes $\left(\mathrm{h}^{+}\right)$were the main active radicals during photocatalysis and hydroxyl radicals $(\mathrm{OH})$ were the secondary radicals. (Mohammed et al. 2021).

$200 \mathrm{mg} / \mathrm{L}$ of ternary composite $\alpha-\mathrm{SiW} 11 \mathrm{Cr} /$ polyaniline $/ \mathrm{ZnO}$ on $10 \mathrm{mg} / \mathrm{L}$ malachite green dye solution was used to check the $\mathrm{pH}$ effect on degradation rate. When the acidity of the solution was increased up to $\mathrm{pH} 1$, the degradation rate was $90.8 \%$ due to the formation of cation ite (CZP) (Reprinted from Mohammed et al. 2021 with permission from Elsevier). HOMO: highest occupied molecular orbital; LUMO: lowest unoccupied molecular orbital; PANI: polyaniline; $\mathrm{ZnO}$ : zinc oxide; $\mathrm{Cu}_{2} \mathrm{O}$ : copper (I) oxide; $\mathrm{CB}$ : conduction band; VB: valence band; $\mathrm{CZP}: \mathrm{Cu}_{2} \mathrm{O} / \mathrm{ZnO} /$ polyaniline ternary composite

form of malachite green which was in favor of $\alpha-\mathrm{SiW} 11 \mathrm{Cr} /$ polyaniline/ $\mathrm{ZnO}$ negatively charged form. The degradation rate was found lowest at $5 \mathrm{pH}$, while the decolorization rate increased at $\mathrm{pH}>7$ because of the fusion of active hydroxide ion and malachite green cation in an aqueous solution. This combination resulted in a colorless alcohol base in basic condition (Zhang et al. 2020).

Some copolymers of conducting polymers were also hybridized with $\mathrm{ZnO}$ for degradation of dyes such as poly(aniline-co-o-aminophenol)/ZnO (Sivakumar et al. 2014), while poly(aniline-co-o-anthranilic acid)/ZnO was used for removal of $\mathrm{Cr}(\mathrm{VI})$ and $\mathrm{Ni}(\mathrm{II})$ and the pattern of reduction was well matched with a first-order kinetic model. The same nanocomposites also showed good results for the degradation of MB dye up to $82 \%$ in UVlight illumination (Haldorai et al. 2014). For the reduction of $\mathrm{Cr}(\mathrm{VI})$, some other researchers also used poly(anilineco-o-toluidine) nanohybrids (Sivakumar et al. 2015). $100 \%$ degradation of Congo red dye was reported by Zor et al. (Sibel and Budak 2020) by polyaniline /ZnO nanohybrids prepared by chemical polymerization, and degradation studies were performed under ultraviolet-visible light irradiation. 


\section{Polyphenylenediamine/zinc oxide}

Polyphenylenediamines are the derivatives of polyaniline and exist in 3 isomers; ortho, meta, and para (Riaz et al. 2017, 2018; Jadoun et al. 2021d). Polyphenylenediamines have two free amine groups for functionalization before and after polymerization to enhance the properties of the polymer such as electroactivity, conductivity, sensitivity, thermal stability for their application in metal ions adsorption, anticorrosion coatings, sensors (Jadoun et al. 2021d), but the nanohybrids of polyphenylenediamines with $\mathrm{ZnO}$ are very rarely studied for photocatalysis (Jadoun et al. 2017a; Riaz et al. 2019; Sandoval et al. 2019). The bandgap of poly(ophenylenediamine) (ortho isomer) is $2.2 \mathrm{eV}$ which is quite low in comparison to $\mathrm{ZnO}$ (Olgun and Gülfen 2014). The photocatalytic activity of homopolymer was very poor under ultraviolet irradiation for the degradation of acid red 249 but the poly(m-phenylenediamine)/ZnO (1/80) (meta isomer) showed the highest degradation of $94.6 \%$ in $120 \mathrm{~min}$. The other ratios and $\mathrm{ZnO}$ showed less activity than the previous one which was $88.9 \%$ for poly(m-phenylenediamine)/ZnO (1/60), $92.3 \%$ for poly(m-phenylenediamine)/ZnO (1/100), and $81.8 \%$ for pristine $\mathrm{ZnO}$, as shown in Fig. 7a. Therefore, increasing the poly (m-phenylenediamine) content in nanohybrids increased the electron mobility due to conjugate structure and recommend the photogenerated electrons and holes recombination, but the intensity of ultraviolet light absorbed by $\mathrm{ZnO}$ particles was affected by more increment in poly(m-phenylenediamine) content.
Under visible light irradiation, both $\mathrm{ZnO}$ and poly(mphenylenediamine) showed poor results with almost no degradation of acid red 249 dye. Although when PMPD/ZnO was irradiated with visible light, poly(m-phenylenediamine) absorbed photons to excite and electrons transferred to conduction band of $\mathrm{ZnO}$, hence charge separation was improved along with photocatalytic activity too and the best results were shown by poly(m-phenylenediamine)/ZnO (1/80), Fig. 7b. The best nanohybrid was studied for photocatalytic stabilities after some cycles with the $50 \mathrm{mg}$ of catalyst and $50 \mathrm{mg} / \mathrm{L}$ concentration of acid red 249 . Photocatalyst was restored by centrifuge after each run and used for the degradation of acid red 249 under ultraviolet and visible light, Fig. 7c, d. After the 5th cycle, $79.4 \%$ acid red 249 was degraded by poly(m-phenylenediamine)/ZnO (1/80) which was $34.9 \%$ in the case of $\mathrm{ZnO}$. This was due to inhibition of photo-corrosion in the presence of poly ( $\mathrm{m}$-phenylenediamine) (Peng et al. 2014). Poly (aniline-co-p-phenylenediamine)/ZnO nanohybrids were found more electrically conductive, thermally stable as compared to pristine copolymer, and a favored interaction between the $\mathrm{ZnO}$ nanoparticle and copolymer chains. These showed $80 \%$ degradation of methylene blue in $3 \mathrm{~h}$ under ultraviolet irradiation (Sivakumar et al. 2012).

\section{Polypyrrole/zinc oxide}

Polypyrrole is a widely explored conducting polymer having a bandgap of $2.2 \mathrm{eV}$ (Jadoun et al. 2021a). The nanohybrids
Fig. 7 The degradation efficiency of acid red 249 versus irradiation time under a ultraviolet irradiation b visible light irradiation $\mathbf{c}$ using poly(m-phenylenediamine)/ zinc oxide (1/80) composite as photocatalyst under ultraviolet irradiation at different recycling runs (the degradation efficiency of acid red 249 after $120 \mathrm{~min}$ irradiation in ultraviolet light at several recycling experiments is shown in inset) $\mathbf{d}$ using poly(m-phenylenediamine)/ zinc oxide (1/80) composite as photocatalyst under visible light irradiation at several recycling runs (Reprinted from Peng et al. 2014 with permission from Wiley)
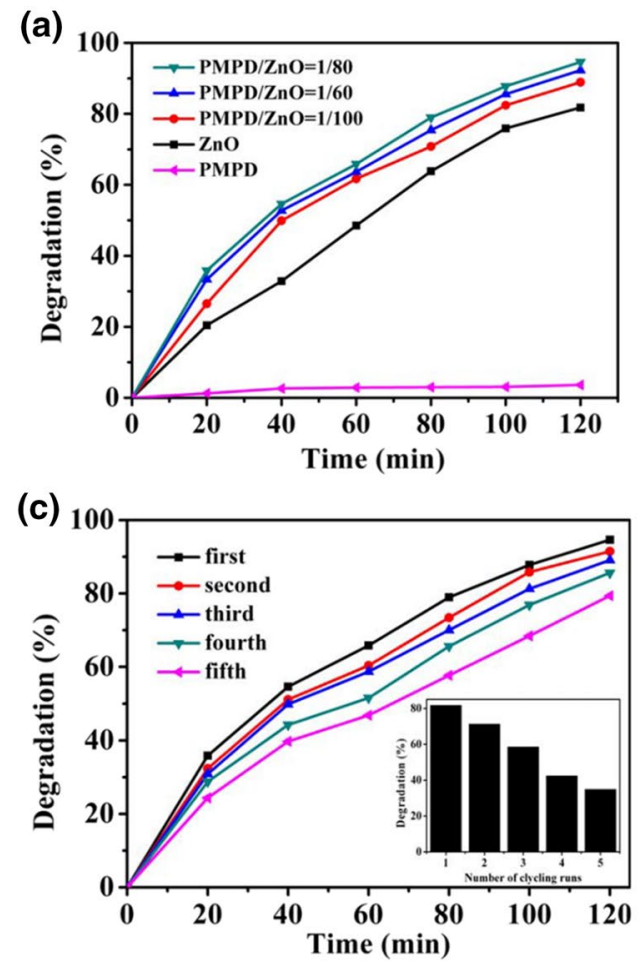
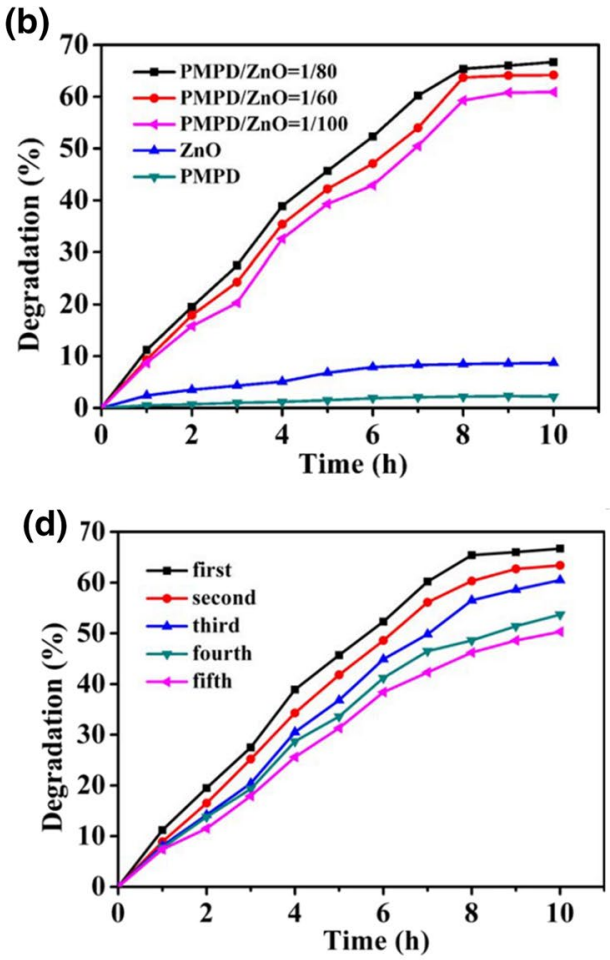
of polypyrrole $/ \mathrm{ZnO}$ possessed 2 times higher rate constant than pristine $\mathrm{ZnO}$ for the degradation of diclofenac which is a non-steroidal anti-inflammatory drug extensively used as analgesics, resulting in $81 \%$ degradation in $60 \mathrm{~min}$. $\mathrm{ZnO}$ alone was able to degrade $46 \%$ of diclofenac only. In 25:1 of polypyrrole: $\mathrm{ZnO}$ showed the best results while increasing the $\mathrm{ZnO}$ content (polypyrrole: $\mathrm{ZnO} / 5: 1$ ) suppressed the surface of polypyrrole resulting in defects in which $\mathrm{e}^{-} / \mathrm{h}^{+}$ recombination occurred and revealed the photocatalytic activity to be decreased. The degradation of diclofenac can be seen in Fig. 8a, with increasing the time absorbance peak of diclofenac decreased suggested $81 \%$ degradation in $60 \mathrm{~min}$. In the degradation of diclofenac, the main responsible species were trapped as $\mathrm{h}^{+}$in the scavenger test (Silvestri et al. 2019). Polypyrrole/ZnO prepared by ultrasonication technique was effective to reduce $\mathrm{Cr}^{6+}$ to $\mathrm{Cr}^{3+}$ under visible light irradiation. The electron moved in conduction band of $\mathrm{ZnO}$ participated in the reduction of $\mathrm{Cr}^{+6}$ to $\mathrm{Cr}^{+3}$ while the holes form hydroxide radical by absorbing water molecules on the surface but it could again oxidize $\mathrm{Cr}^{+3}$ to $\mathrm{Cr}^{+6}$. Hence, the addition of citric acid as a radicle scavenging agent was done to enhance to photoreduction of $\mathrm{Cr}^{+6}$ and inhibit the oxidation process, Fig. $8 \mathrm{~b}$ (Balakumar and Baishnisha 2021).

A flexible composite film of ZnO-Micro-rods/ polypyrrole with substantial durability, flexibility, and photocatalytic activity under visible light was developed in which $\mathrm{ZnO}$ microrods were coated with polypyrrole shell ( $400 \mathrm{~nm}$ thick) from the upper part and acted as a photosensitizer, while the lower part of $\mathrm{ZnO}$ was also implanted in polypyrrole layer which enabled the transfer of an electron from LUMO of polypyrrole to conduction band of $\mathrm{ZnO}$, Fig. 9a. This

(a)

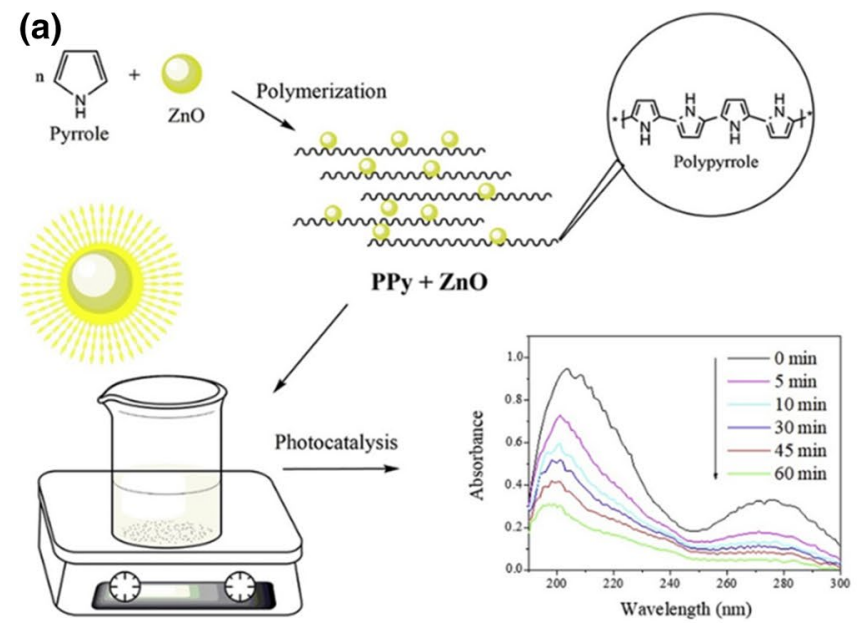

Fig. 8 Nanohybrids of polypyrrole /zinc oxide a Synthesis of polypyrrole/zinc oxide nanocomposites for UV-visible photocatalytic degradation of diclofenac b Photocatalysis by polypyrrole /zinc oxide for reduction of toxic $\mathrm{Cr}^{6+}$ to benign $\mathrm{Cr}^{3+}$ (Reprinted from Silvestri type of architecture enhanced the photocatalytic degradation of MB with a $0.22 \%$ rate per minute, Fig. $9 \mathrm{~b}$ (Yan et al. 2017). Similarly, polypyrrole/ZnO nanohybrids were used to degrade the violet acid 7 dye for treatment of wastewater (González-Casamachin et al. 2019) and polypyrrole /1-D $\mathrm{ZnO}$ nanohybrids showed $97.13 \%$ degradation of crystal violet using $1 \mathrm{gL}^{-1}$ of catalyst and $5 \mathrm{mgL}^{-1}$ concentration of dye in $120 \mathrm{~min}$ (PATIL et al. 2016). Nanohybrids synthesized by polypyrrole, $\mathrm{ZnO}$, and $\mathrm{Ag}$ were studied for the degradation of Rhodamine B resulted in $99 \%$ of dye degradation in 120 min under visible light irradiation with a rate constant of $\mathrm{k}=2.96 \times 10^{-2} \mathrm{~min}^{-1}$ (Podasca et al. 2019).

The real untreated textile water containing azo dye direct black 22 was initially treated with the bacterial consortium and after $96 \mathrm{~h}$ polypyrrole/ZnO nanohybrids were employed for photocatalysis. The photocatalysis was carried out for $60 \mathrm{~min}$ and revealed $95.7 \%$ of decolorization efficiency and $99.8 \%$ of total organic carbon degradation. Hence, this can be an efficient alternative to put on before dumping textile wastes in water bodies (Ceretta et al. 2020).

\section{Poly (ethylene dioxythiophene)/zinc oxide}

Poly (3,4-ethylene dioxythiophene) is a conducting polymer and has been studied widely for its stability, high and tunable electrical conductivity. In its conducting state, this polymer remains optically transparent, with low redox potential and a moderate bandgap (Gueye et al. 2020). Ternary nanohybrids, poly (ethylene dioxythiophene)/ZnO/fly ash cenosphere (fly ash cenosphere) are very low-density materials which generally used to prepare very lightweight hybrids) was prepared by chemical oxidative polymerization method and utilized

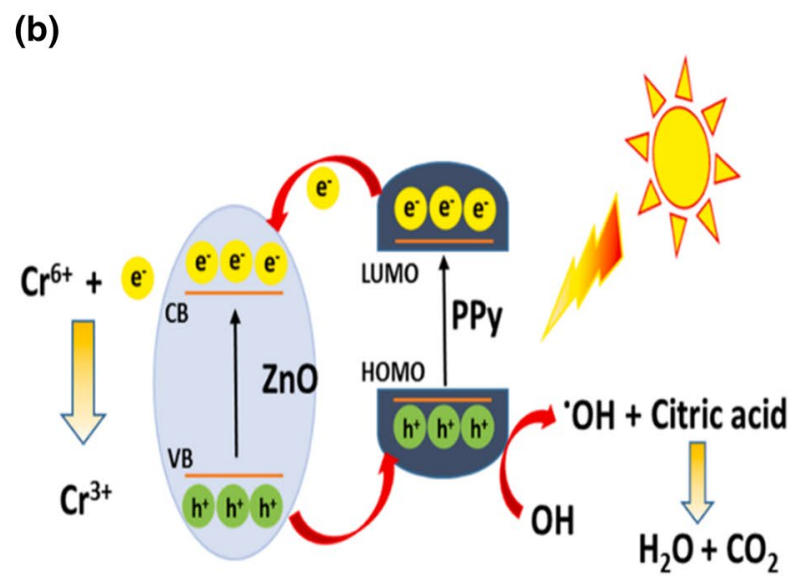

et al. 2019, (Balakumar and Baishnisha 2021) with permission from Elsevier). PPY: polypyrrole; ZnO: zinc oxide; HOMO: highest occupied molecular orbital; LUMO: lowest unoccupied molecular orbital 
(a)

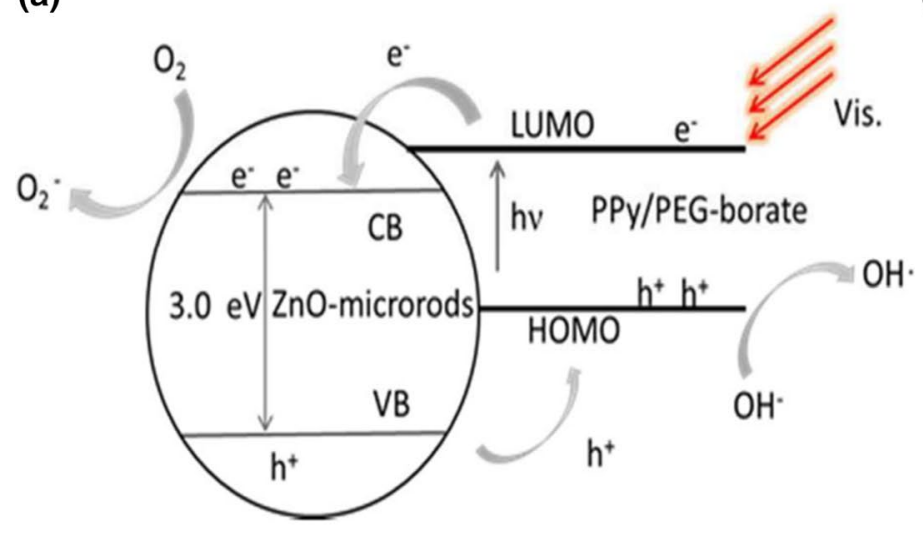

(b)

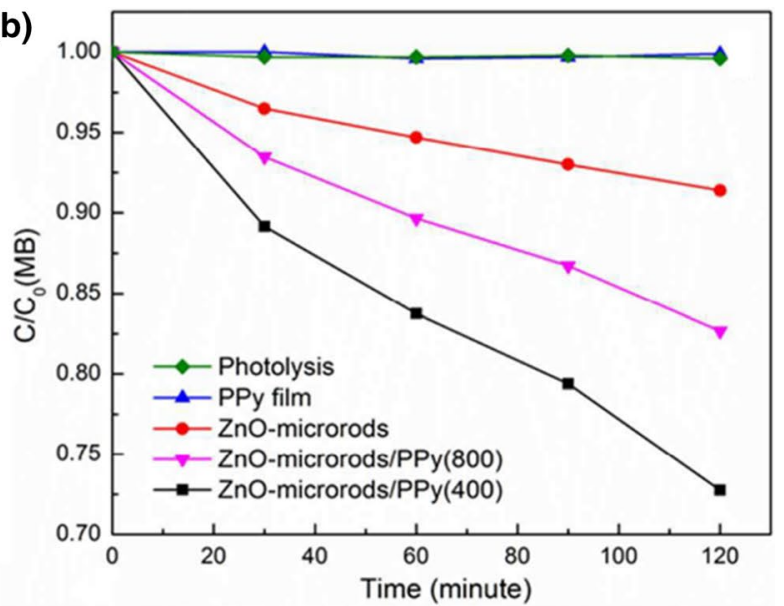

Fig. 9 Photocatalytic activity and mechanism of carrier transport. a Degradation rates of methylene blue by photolysis, polypyrrole/ polyethyleneglycol-borate film, zinc oxides microrod arrays, zinc oxide microrods/ polypyrrole (400) composite film, and zinc oxidemicrorods/ polypyrrole (800) composite film under visible light. b A schematic illustration of carrier separation and transport mecha-

for the photodegradation of methyl orange dye consisting of $\mathrm{N}=\mathrm{N}$ azo group. The photocatalyst with maximum degradation efficiency was $2 \%$ poly (ethylene dioxythiophene)/ $\mathrm{ZnO} /$ fly ash cenosphere composites which showed firstorder kinetics and $0.0058 \mathrm{~min}^{-1}$ rate constant. These ternary nanocomposites were able to degrade $70 \%$ methyl orange dye in 120 min (Dagar and Narula 2016).

A simple solid-state heating method was adopted for the fabrication of poly (ethylene dioxythiophene)/ZnO nanohybrids with the variation of $\mathrm{ZnO}$ content from 10 to $20 \%$. Its photocatalytic activity was measured against the methylene blue in wastewater under UV and solar irradiation. After $5 \mathrm{~h}$ of irradiation under ultraviolet light, the degradation efficiency was found $88.7 \%$ (poly (ethylene dioxythiophene) $/ 10$ $\mathrm{wt} \% \mathrm{ZnO}$ ), $98.7 \%$ (poly (ethylene dioxythiophene) $/ 15$ $\mathrm{wt} \% \mathrm{ZnO}$ ) and $98.2 \%$ (poly (ethylene dioxythiophene) $/ 20$ $\mathrm{wt} \% \mathrm{ZnO}$ ), while in natural sunlight, 93.3\% (poly (ethylene dioxythiophene)/10wt\% $\mathrm{ZnO}$ ), $96.6 \%$ (poly (ethylene dioxythiophene) $/ 15 \mathrm{wt} \% \mathrm{ZnO}$ ) and $95.4 \%$ (poly (ethylene dioxythiophene) $/ 20 \mathrm{wt} \% \mathrm{ZnO}$ ) were achieved which indicated that highest degradation was achieved in ultraviolet light for $15 \mathrm{wt} \%$ of $\mathrm{ZnO}$ in poly (ethylene dioxythiophene)/ $\mathrm{ZnO}$ (Abdiryim et al. 2014).

For the treatment of reactive red 45 azo dye from wastewater, these nanohybrids were prepared by in situ methods and were used under ultraviolet-A and solar light irradiation, and surprisingly very low amount of photocatalyst was able to degrade the dye during wastewater treatment. Before starting the treatment, reactive red 45 dye was kept for maintaining equilibrium. In ultraviolet-A light the photocatalysis was done for $90 \mathrm{~min}$ with the initial concentration of catalysts at nism at the zinc oxide/ polypyrrole interface. (Reprinted from Yan et al. 2017 with permission from American Chemical Society). PPY: polypyrrole; PEG: polyethylene glycol; MB: methylene blue; $\mathrm{ZnO}$ : zinc oxide; HOMO: highest occupied molecular orbital; LUMO: lowest unoccupied molecular orbital

$1 \mathrm{~g} \mathrm{~L}^{-1}$ and $30 \mathrm{mg} \mathrm{L}^{-1}$ for the reactive red 45 dye. Poly (ethylene dioxythiophene)/ZnO (1-2) showed the best results as only $25 \%$ dye was left in $90 \mathrm{~min}$ while in the case of poly (ethylene dioxythiophene)/ZnO (1-3) and poly (ethylene dioxythiophene)/ZnO (1-5), $82 \%$ and $91 \%$ remained in the water, respectively. In the solar irradiation, the results were not satisfying that much as the highest degradation was achieved by poly (ethylene dioxythiophene)/ZnO (1-2) and 53\% dye was remained in the water after 90 min (Katančić et al. 2017).

\section{Other conducting polymers /zinc oxide nanohybrids}

Polynaphthylamine is a derivative of polyaniline (Jadoun et al. 2017c) and much work has been reported previously on polynaphthylamine for photocatalytic activity (Riaz and Ashraf 2011, 2012). Its nanohybrids with $\mathrm{ZnO}$ were prepared by blending polynaphthylamine and $\mathrm{ZnO}$ via sonication for $2 \mathrm{~h}$ and then washed and dried to yield in powder form. These nanohybrids were used to degrade alizarin red dye by two distinct methods (1) photochemical reactor (2) microwave-assisted degradation. For degradation studies, alizarin red dye solution in a fixed concentration was taken for both microwave and ultraviolet irradiation. Firstly, the neat dye solution was exposed in both for $5,10,20,30$, and $40 \mathrm{~min}$ in the microwave while $30,60,90,120 \mathrm{~min}$ for ultraviolet irradiation. The same process was repeated by adding $200 \mathrm{mg}$ of $\mathrm{ZnO}$, polynaphthylamine, and polynaphthylamine / $\mathrm{ZnO}$ nanohybrids. As well as the time increased, the absorption maxima of the peak of dye decreased revealed the degradation of dye, and the maximum decrease in the visible 


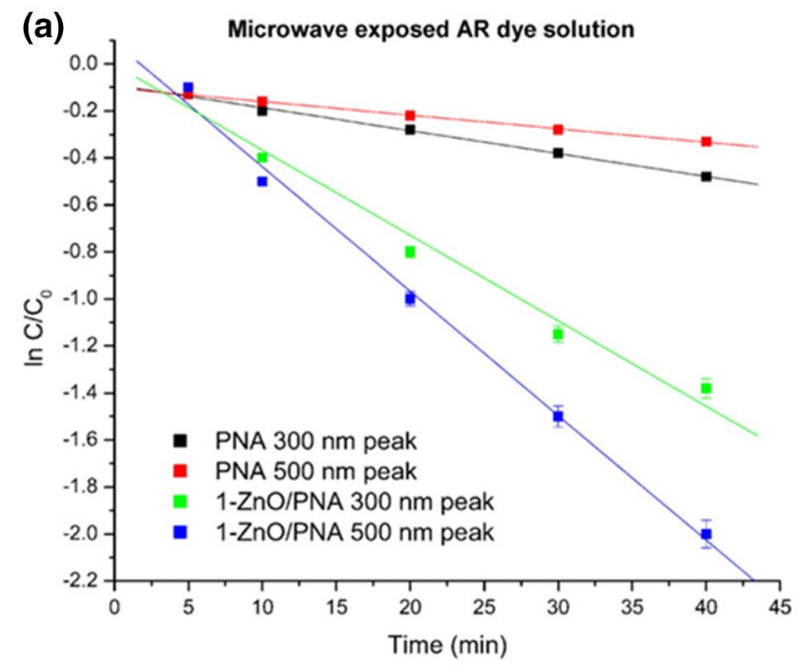

(c)

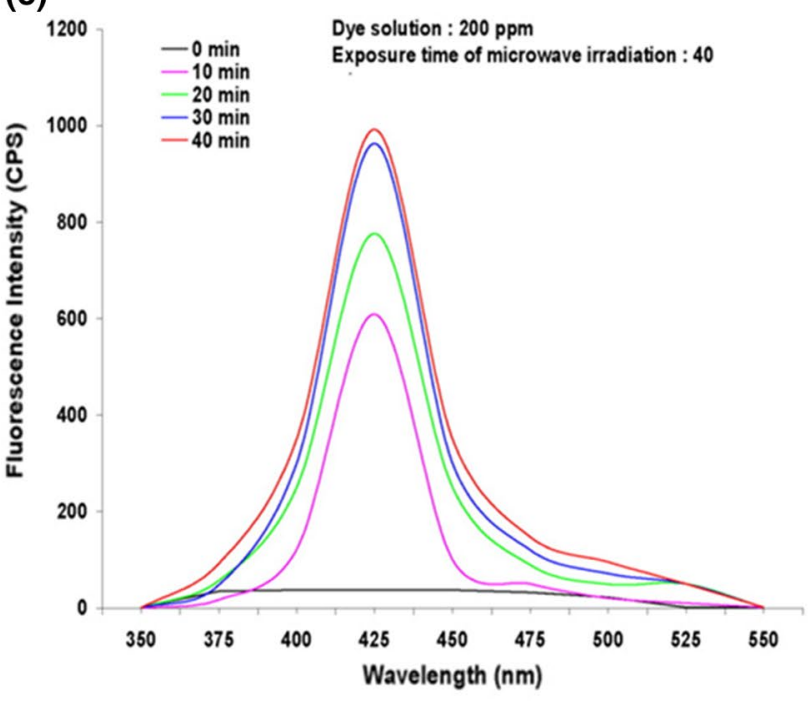

Fig. 10 Degradation kinetic plot for alizarin red dye solution containing homopolymer and nanohybrid of polynaphthylamine/zinc oxide as catalyst exposed to a microwave irradiation $\mathbf{b}$ ultraviolet irradiation

region was found in the case of microwave irradiation. The degradation kinetics showed first order in both cases, and the $\mathrm{k}$ values in ultraviolet exposure were found 0.005 for $300 \mathrm{~nm}$ peak while 0.004 for $500 \mathrm{~nm}$ peak, Fig. 10a, b. In the case of microwave irradiation, these were 0.009 and 0.007 , respectively, for ultraviolet and visible region peak, and rate constants also followed the same pattern. In the degradation pathway, $\mathrm{OH}$ was generated in both microwave and ultraviolet degradation. Terephthalic acid was used to trap $\mathrm{OH}$ as it vastly reacts with the previous one to produce 2-hydroxyterephthalic acid which is highly fluorescent and gives emission on $415 \mathrm{~nm}$ with an excitation at $315 \mathrm{~nm}$. The increment in peak intensity of 2-hydroxyterephthalic

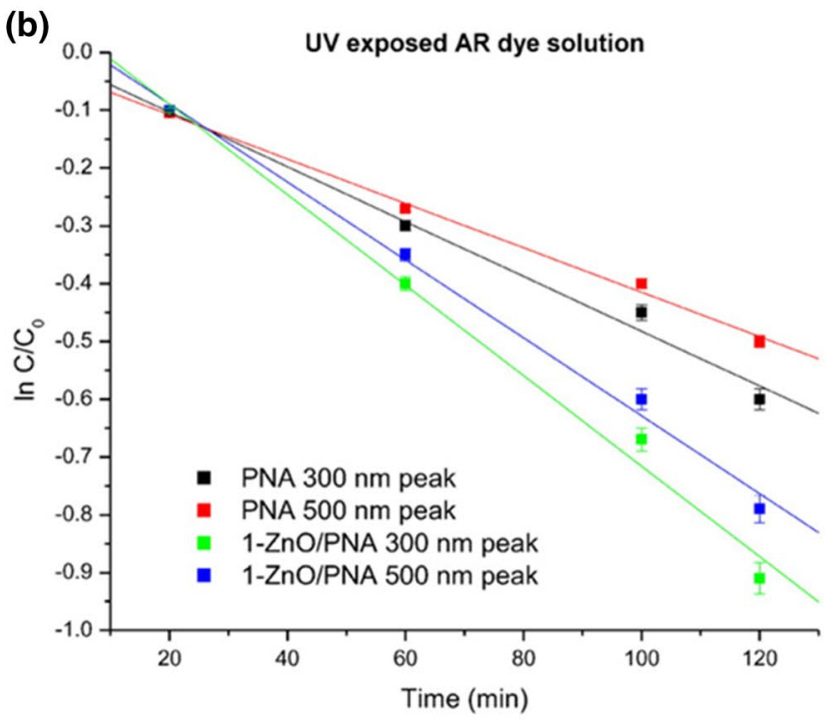

(d)

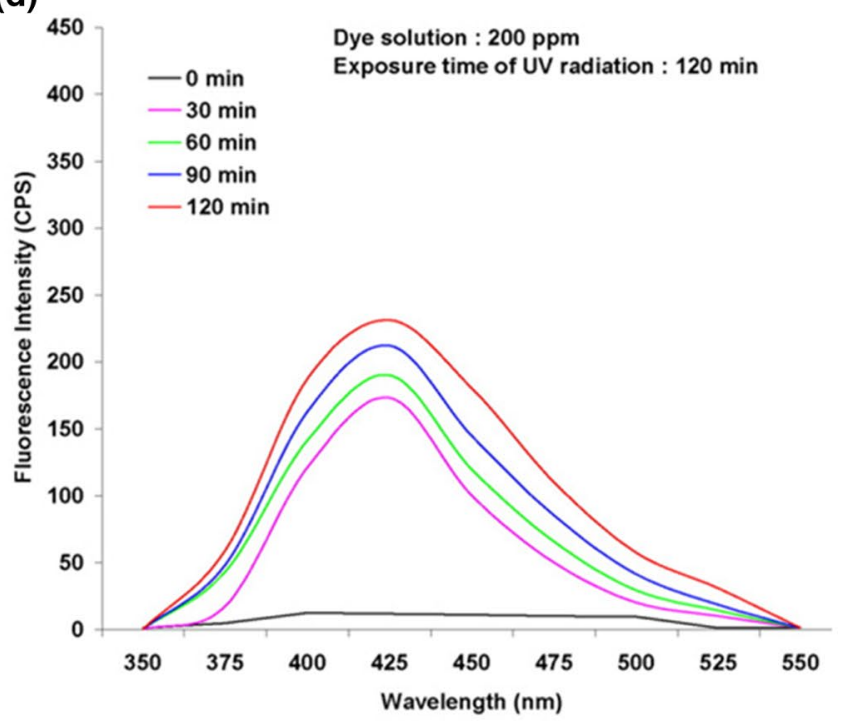

c variation of fluorescence intensity of 2-hydroxy terephthalic acid upon microwave irradiation d ultraviolet irradiation (Reprinted from Riaz et al. 2016 with permission from Elsevier)

acid with time under microwave and ultraviolet but surprisingly, the peak intensity was increased more than twice in case of the microwave which indicated the generation of $\mathrm{OH}$ in microwave irradiation was high, Fig. 10c, d (Riaz et al. 2016).

The photocatalytic degradation of methylene blue under visible light was performed by polynaphthylamine/ZnO synthesized via in situ chemical oxidative polymerization. The surface properties were modified by adding $\mathrm{ZnO}$ to polynaphthylamine as well as the morphology and crystallinity were also found to enhance. The degradation rate was moderate and up to $22 \%$ dye was degraded under visible light irradiation by polynaphthylamine $/ 0.39 \mathrm{wt} \% \mathrm{ZnO}$. 
Polythiophene is an intrinsically conducting polymer having sulfur in its cycle and conjugated double bonds in its backbone. It attracted a lot of attention to researchers due to its good thermal stability, higher environmental stability, and narrow bandgap (2.5 eV) (Swager 2017). In nanohybrids of polythiophene $/ \mathrm{ZnO}$, the photons are easily absorbed by polythiophene upon irradiation with visible light, but still, it is very less studied with $\mathrm{ZnO}$. Different weight ratios of polythiophene $\mathrm{ZnO}$ were explored for the photocatalytic degradation of MB. Significant decrement in absorbance of methylene blue was attained after some time and almost diminished in $180 \mathrm{~min}$. The charge carriers generated in conduction band and valence band moved toward the catalyst surface were responsible for redox reactions with other moieties. $\mathrm{OH}$ and $\mathrm{O}_{2}{ }^{--}$reacted with methylene blue molecule to break the backbone of dye or pollutant, Fig. 11 (Faisal et al. 2020).

Shivakumar and coworkers fabricated Poly(anilineco-o-toluidine)/ $\mathrm{ZnO}$ nanohybrids for the reduction of $\mathrm{Cr}$ (VI) from wastewater and the patterns of reduction of $\mathrm{Cr}$ (VI) followed the first-order kinetics, as well as these nanohybrids, also showed outstanding degradation of MB. 65\% reduction of $\mathrm{Cr}(\mathrm{VI})$ and $92 \%$ for $\mathrm{MB}$ dye were obtained in $3 \mathrm{~h}$ duration (Sivakumar et al. 2015). The poly(azomethyne)/ $\mathrm{ZnO}$ (poly(azomethyne) is the Schiff's base or conjugated polymer achieved by condensation having conjugated nitrogen atom $(\mathrm{CH}=\mathrm{N})$ in a polymer backbone) was used for the decolorization and photocatalytic degradation of dyes containing wastewater in natural sunlight. The dose of the photocatalyst was taken $500 \mathrm{mg}$, while the dye was taken in $50 \mathrm{mg} \mathrm{L}^{-1}$ concentration. The degradation efficiency was found $97 \%$ for MB and $96 \%, 95 \%$ were found for malachite green and Bismarck brown dyes in $5 \mathrm{~h}$ and the rate constant of pseudo-first-order reaction kinetics was found 0.2803 , 0.3232 , and $0.3762 \mathrm{~min}^{-1}$ for methylene blue, malachite

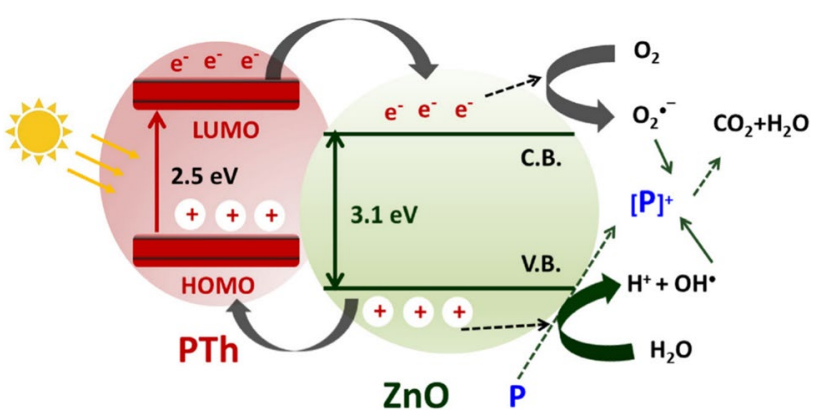

Fig. 11 Photocatalytic degradation of methylene blue dye or gemifloxacin antibiotic by polythiophene/zinc oxide nanohybrid photocatalyst, where $[\mathrm{P}]$ represents the pollutant molecule (Reprinted from Faisal et al. 2020 with permission from Elsevier). PTh: polythiophene; C.B.: conduction band; V.B.: valence band $\mathrm{ZnO}$ : zinc oxide; HOMO: highest occupied molecular orbital; LUMO: lowest unoccupied molecular orbital green, and brilliant blue dyes, respectively (Pradeeba and Sampath 2019).

Various conducting polymers such as polyaniline, poly(ophenylenediamine), polypyrrole, polythiophene, and polynaphthylamine possess a narrower bandgap of $\sim 2-2.5 \mathrm{eV}$, while $\mathrm{ZnO}$ possesses a wide bandgap of $3.37 \mathrm{eV}$, works as a synergistic relationship to each other and minimizes the recombination of charge by the synergistic charge transfer mechanism resulting in enhancement of redox reactions at the surface of catalyst for the removal of toxic pollutants and reduction of metals. Therefore, conducting polymers / $\mathrm{ZnO}$ nanohybrids are the best alternative to absorb the solar irradiation up to $53 \%$ due to its visible region lying bandgap.

\section{Effectiveness of conducting polymers/ zinc oxide photocatalyst with other semiconductors}

Conducting polymers possessed a narrow bandgap of $\sim 2-2.5 \mathrm{eV}$ (Guo and Facchetti 2020), revealing its potential for sensitization of $\mathrm{ZnO}$ having a wide bandgap of $3.2 \mathrm{eV}$ (Khan et al. 2017). The excellent photocatalysis ability of conducting polymers / $\mathrm{ZnO}$ nanohybrids is attributed to the adequate charge separation capability and movement of electrons from HOMO to LUMO of conducting polymers followed by the transfer of electrons to conduction band of $\mathrm{ZnO}$. The electrons from valence band of $\mathrm{ZnO}$ also transferred to conduction band of $\mathrm{ZnO}$; hence, the holes created in valence band transferred to HOMO of conducting polymers. Consequently, on the surface of $\mathrm{ZnO}$, hydroxides and superoxide radicals generate due to the excess of holes in valence band to enhance photocatalysis. These radicals are responsible for the redox reactions and degradation of organic contaminants from wastewater (Belabed et al. 2021; Khan et al. 2021). When compared to pristine poly (ethylene dioxythiophene) and $\mathrm{ZnO}$ with their nanohybrids for photocatalysis of methylene blue under ultraviolet light for $5 \mathrm{~h}$, the efficiency of poly (ethylene dioxythiophene)/ $\mathrm{ZnO}$ was 52\%, while pristine poly (ethylene dioxythiophene) and $\mathrm{ZnO}$ revealed $14 \%$ and $31 \%$, respectively, which were quite low (Ali et al. 2013).

For degradation of methylene blue and malachite green, polyaniline/ZnO nanohybrids showed an affective behavior under visible light illumination. The intermolecular interaction between polyaniline and $\mathrm{ZnO}$ resulted in $\pi-\pi^{*}$ transition of electrons from HOMO to LUMO of polyaniline which transferred in conduction band of $\mathrm{ZnO}$ resulting in hydroxide and superoxide anion radical's generation which were responsible for photocatalysis (Saravanan et al. 2016). The solar light on polyaniline / $\mathrm{ZnO}$ nanohybrids boosted the degradation of dyes up to $99 \%$ in $5 \mathrm{~h}$ (Eskizeybek et al. 2012). The nanohybrids of polyaniline $/ \mathrm{TiO}_{2}$ and polypyrrole $/ \mathrm{TiO}_{2}$ 
were employed for the degradation of methyl orange under solar light resulting in satisfactory results but less degradation efficiency when compared to polyaniline $/ \mathrm{ZnO}$ and polypyrrole $\mathrm{ZnO}$ nanohybrids (Deng et al. 2012; OvandoMedina et al. 2015; Kumar et al. 2018).

\section{Conclusion}

In the nutshell, nanohybrids of conducting polymers and zinc oxide seem to be the most promising materials for photocatalysis under, visible and solar irradiation. Numerous pieces of the literature suggested that minimizing the possibility of recombination of charge carriers is the easiest and effective way for enhancing photocatalytic activity. Therefore, conducting polymer-based zinc oxide nanohybrids is the best alternative in which the bandgap of nanohybrid lies in the visible region which increases the possibility to absorb solar irradiation up to 53\%. This critical review demonstrated the use of conducting polymers and their paradigm shift to enhance the performance of zinc oxide-based nanohybrid photocatalyst for degradation of harmful chemicals, toxic pollutants, insecticides, etc. Along with zinc oxide, the combination of conducting polymers enhances the photogenerated charge carrier separation by extending the absorption of light from UV to the visible region, and hence, the visible-light-driven photocatalytic activity improved. A synergistic relation between conducting polymers and zinc oxide is also responsible for the enhanced efficiency of photocatalytic degradation. As the aggregation process is slowed down by using polymers due to its tendency, the use of conducting polymer during synthesis in high concentration is much helpful in the reduction of large aggregation in photocatalysis. Photoinduced holes can be easily trapped by $\pi$-conjugated chains of conducting polymers, supportive in inhibiting the self-oxidation process of photocatalysts hinders the photo-corrosion resulting in improving the photostability.

Though more than $90 \%$ of studies are related to the degradation of dyes, in reality, the removal of dye is a simpler process than some complex emerging organic contaminants such as personal care products, disinfection by-products, pesticides, drugs, and many other synthetic chemicals which should be more focused. Also, the photocatalysis on conducting polymers/ZnO nanohybrids and all other nanohybrids has been researched on a static system which is hard to apply on the water treatment in the real-world system due to the need for sustainability of catalyst in a flow-through system with the slight leakage of metals from the nanohybrids. Future soundings should be the study of the removal of major contaminants from real water systems by simulating the quality parameters from laboratory to field. The posttreatment retrieval of nanohybrids must be majorly focused to scale it up. Some magnetic separation systems should be the key point for these types of post-treatment for recovery of nanohybrids so that by applying a magnetic field, these could be easily separated out.

In the field of solar energy harvesting and storage, photocatalysis can open new opportunities and there is wide space for development. Even, it can play a key role also in environmental diminutions by some routes to remove several types of organic and inorganic pollutants which are inorganic toxins such as arsenic, chromium, mercury and others in the freshwater system as well as for the reduction and harvesting of copper or noble metals. Some better new engineering concepts should be optimized for new materials for photocatalysis. To attain better solar radiation, there is a need to fabricate those materials which are shifting to the visible and near-infrared region as $43 \%$ of sunlight absorb in the visible region and $52 \%$ in the near-infrared region. In parallel with photocatalytic experiments, environmental toxicity valuation should also be carried out. All in all, conducting polymer/zinc oxide-based photocatalysis has shown a great potential to remove organic contaminants in laboratory-scale settings, however, its application in the real world would necessitate addressing various hurdles which should be focused on in the future.

Acknowledgements The authors are grateful for the support of the National Research and Development Agency of Chile (ANID) and the projects, FONDECYT Postdoctoral 3200850, FONDECYT 1191572 and ANID/FONDAP/15110019. The authors are also thankful to Elsevier, Springer, American Chemical Society, Taylor \& Francis, and MDPI for copyright permission

Funding The authors have not disclosed any funding

\section{Declarations}

Conflict of interest The author declares no conflict of interest.

\section{References}

Abdiryim T, Ali A, Jamal R et al (2014) A facile solid-state heating method for preparation of poly(3,4-ethelenedioxythiophene)/ZnO nanocomposite and photocatalytic activity. Nanoscale Res Lett 9:89. https://doi.org/10.1186/1556-276X-9-89

Abinaya M, Rajakumaran R, Chen S-M et al (2019) In Situ synthesis, characterization, and catalytic performance of polypyrrole polymer-incorporated $\mathrm{Ag}_{2} \mathrm{MoO}_{4}$ nanocomposite for detection and degradation of environmental pollutants and pharmaceutical drugs. ACS Appl Mater Interfaces 11:38321-38335. https:// doi.org/10.1021/acsami.9b13682

Acuña K, Yáñez J, Ranganathan S et al (2017) Photocatalytic degradation of roxarsone by using synthesized $\mathrm{ZnO}$ nanoplates. Sol Energy. 157:335-341. https://doi.org/10.1016/j.solener.2017.07. 054

Adabavazeh H, Saljooqi A, Shamspur T, Mostafavi A (2021) Synthesis of polyaniline decorated with $\mathrm{ZnO}$ and $\mathrm{CoMoO}_{4}$ nanoparticles 
for enhanced photocatalytic degradation of imidacloprid pesticide under visible light. Polyhedron 198:115058. https://doi.org/ 10.1016/j.poly.2021.115058

Ahmad N, Sultana S, Faisal SM et al (2019) Zinc oxide-decorated polypyrrole/chitosan bionanocomposites with enhanced photocatalytic, antibacterial and anticancer performance. RSC Adv 9:41135-41150. https://doi.org/10.1039/C9RA06493A

Akpomie KG, Conradie J (2020) Banana peel as a biosorbent for the decontamination of water pollutants. A Review Environ Chem Lett 18:1085-1112. https://doi.org/10.1007/s10311-020-00995-x

Albiter E, Merlano AS, Rojas E et al (2021) Synthesis, characterization, and photocatalytic performance of $\mathrm{ZnO}-$ Graphene nanocomposites: a review. J Compos Sci 5:4. https://doi.org/10.3390/ jes5010004

Ali A, Jamal R, shao W, et al (2013) Structure and properties of solidstate synthesized poly(3,4-propylenedioxythiophene)/nano-ZnO composite. Prog Nat Sci Mater Int 23:524-531. https://doi.org/ 10.1016/j.pnsc.2013.11.002

Ambalagi SM, Inamadar HK, Sannakki B (2016) Mechanism of DC conductivity measurement of zinc oxide doped polyaniline nanocomposites. Mater Today Proc 3:3945-3950. https://doi.org/10. 1016/j.matpr.2016.11.054

Ameen S, Akhtar MS, Kim YS et al (2010) Synthesis and characterization of novel poly(1-naphthylamine)/zinc oxide nanocomposites: application in catalytic degradation of methylene blue dye. Colloid Polym Sci 288:1633-1638. https://doi.org/10.1007/ s00396-010-2284-9

Ameen S, Akhtar MS, Kim YS et al (2011) An effective nanocomposite of polyaniline and $\mathrm{ZnO}$ : preparation, characterizations, and its photocatalytic activity. Colloid Polym Sci 289:415-421. https:// doi.org/10.1007/s00396-010-2350-3

Amita M, Kiruthiga K, Mahalakshmi S et al (2019) Structural, microstructural, electrical, thermal and non-isothermal degradation kinetic studies on technologically important poly (aniline)/CdO nanocomposites. J Sol-Gel Sci Technol 91:611-623. https://doi. org/10.1007/s10971-019-05060-w

Asgari E, Esrafili A, Jafari AJ et al (2019) The comparison of $\mathrm{ZnO} /$ polyaniline nanocomposite under UV and visible radiations for decomposition of metronidazole: degradation rate, mechanism and mineralization. Process Saf Environ Prot 128:65-76. https:// doi.org/10.1016/j.psep.2019.05.050

Balakumar V, Baishnisha A (2021) Rapid visible light photocatalytic reduction of $\mathrm{Cr}^{6+}$ in aqueous environment using $\mathrm{ZnO}$-PPy nanocomposite synthesized through ultrasonic assisted method. Surfaces and Interfaces. 23:100958. https://doi.org/10.1016/j.surfin. 2021.100958

Belabed C, Tab A, Bellal B et al (2021) High photocatalytic performance for hydrogen production under visible light on the hetero-junction Pani-ZnO nanoparticles. Int J Hydrogen Energy 46:17106-17115. https://doi.org/10.1016/j.ijhydene.2021.02.165

Bera S, Won D-I, Rawal SB et al (2019) Design of visible-light photocatalysts by coupling of inorganic semiconductors. Catal Today 335:3-19. https://doi.org/10.1016/j.cattod.2018.11.001

Bhavya G, Belorkar SA, Mythili R et al (2021) Remediation of emerging environmental pollutants: a review based on advances in the uses of eco-friendly biofabricated nanomaterials. Chemosphere 275:129975. https://doi.org/10.1016/j.chemosphere.2021.129975

Bomila R, Suresh S, Srinivasan S (2019) Synthesis, characterization and comparative studies of dual doped $\mathrm{ZnO}$ nanoparticles for photocatalytic applications. J Mater Sci Mater Electron 30:582592. https://doi.org/10.1007/s10854-018-0324-2

Ceretta MB, Vieira Y, Wolski EA et al (2020) Biological degradation coupled to photocatalysis by $\mathrm{ZnO} /$ polypyrrole composite for the treatment of real textile wastewater. J Water Process Eng. 35:101230. https://doi.org/10.1016/j.jwpe.2020.101230
Chauhan NPS, Mozafari M, Chundawat NS et al (2016) High-performance supercapacitors based on polyaniline-graphene nanocomposites: Some approaches, challenges and opportunities. J Ind Eng Chem. 36:13-29. https://doi.org/10.1016/j.jiec.2016.03.003

Crini G (2021) Cyclodextrin-epichlorohydrin polymers synthesis, characterization and applications to wastewater treatment: a review. Environ Chem Lett. https://doi.org/10.1007/ s10311-021-01204-Z

da Nascimento KN, S, de Oliveira MCA, Oliveira PS, et al (2015) Photocatalytic activity of $\mathrm{ZnO}$ composites against rhodamine $\mathrm{B}$ and rhodamine 6G. Fibers Polym 16:2177-2183. https://doi.org/ 10.1007/s12221-015-5456-x

Dagar A, Narula AK (2016) Effect of ternary PEDOT/ZnO/Flyashcenosphere photocatalyst on photo-degradation of methyl orange under visible light. J Mater Sci Mater Electron 27:12777-12785. https://doi.org/10.1007/s10854-016-5410-8

Demir MM, Memesa M, Castignolles P, Wegner G (2006) PMMA/zinc oxide nanocomposites prepared by in-situ bulk polymerization. Macromol Rapid Commun 27:763-770. https://doi.org/10.1002/ marc. 200500870

Deng F, Li Y, Luo X et al (2012) Preparation of conductive polypyrrole/TiO2 nanocomposite via surface molecular imprinting technique and its photocatalytic activity under simulated solar light irradiation. Colloids Surfaces A Physicochem Eng Asp. 395:183-189. https://doi.org/10.1016/j.colsurfa.2011.12.029

Deng X, Chen Y, Wen J et al (2020) Polyaniline-TiO 2 composite photocatalysts for light-driven hexavalent chromium ions reduction. Sci Bull 65:105-112. https://doi.org/10.1016/j.scib.2019.10.020

Durgalakshmi D, Rakkesh RA, Rajendran S, Naushad M (2020) Principles and Mechanisms of Green Photocatalysis. In: Green Photocatalysts. Springer, pp 1-24. https://doi.org/10.1007/ 978-3-030-15608-4_1

Eskizeybek V, Sarı F, Gülce H et al (2012) Preparation of the new polyaniline/ZnO nanocomposite and its photocatalytic activity for degradation of methylene blue and malachite green dyes under UV and natural sun lights irradiations. Appl Catal B Environ 119-120:197-206. https://doi.org/10.1016/j.apcatb.2012.02.034

Faisal M, Harraz FA, Jalalah M et al (2020) Polythiophene doped ZnO nanostructures synthesized by modified sol-gel and oxidative polymerization for efficient photodegradation of methylene blue and gemifloxacin antibiotic. Mater Today Commun 24:101048. https://doi.org/10.1016/j.mtcomm.2020.101048

Ferreira DC, Graziele I, Marques RC, Gonçalves J (2021) Investment in drinking water and sanitation infrastructure and its impact on waterborne diseases dissemination: The Brazilian case. Sci Total Environ 779:146279. https://doi.org/10.1016/j.scitotenv. 2021.146279

Fujishima A, Honda K (1972) Electrochemical photolysis of water at a semiconductor electrode. Nature 238:37-38. https://doi.org/ $10.1038 / 238037 \mathrm{a} 0$

Fujishima A, Rao TN, Tryk DA (2000) Titanium dioxide photocatalysis. J Photochem Photobiol C Photochem Rev 1:1-21. https://doi. org/10.1016/S1389-5567(00)00002-2

Fujishima A, Zhang X, Tryk DA (2008) $\mathrm{TiO}_{2}$ photocatalysis and related surface phenomena. Surf Sci Rep 63:515-582. https:// doi.org/10.1016/j.surfrep.2008.10.001

Ghosh S (2021) Conjugated polymer nanostructures for photocatalysis. Conjug Polym Nanostructures Energy Convers Storage Appl. https://doi.org/10.1002/9783527820115

Gilja V, Vrban I, Mandić V et al (2018) Preparation of a PANI/ZnO composite for efficient photocatalytic degradation of acid blue. Polymers (basel). https://doi.org/10.3390/polym10090940

González-Casamachin DA, Rivera De la Rosa J, Lucio-Ortiz CJ et al (2019) Visible-light photocatalytic degradation of acid violet 7 dye in a continuous annular reactor using $\mathrm{ZnO} / \mathrm{PPy}$ photocatalyst: Synthesis, characterization, mass transfer effect evaluation and 
kinetic analysis. Chem Eng J 373:325-337. https://doi.org/10. 1016/j.cej.2019.05.032

Gueye MN, Carella A, Faure-Vincent J et al (2020) Progress in understanding structure and transport properties of PEDOT-based materials: a critical review. Prog Mater Sci 108:100616. https:// doi.org/10.1016/j.pmatsci.2019.100616

Guo X, Facchetti A (2020) The journey of conducting polymers from discovery to application. Nat Mater 19:922-928. https://doi.org/ 10.1038/s41563-020-0778-5

Haldorai Y, Sivakumar K, Shim J-J (2014) ZnO nanoparticles dispersed poly(aniline-co-o-anthranilic acid) composites: Photocatalytic reduction of $\mathrm{Cr}(\mathrm{VI})$ and $\mathrm{Ni}(\mathrm{II})$. Polym Compos 35:839-846. https://doi.org/10.1002/pc.22727

Hashimoto K, Irie H, Fujishima A (2005) $\mathrm{TiO}_{2}$ photocatalysis: a historical overview and future prospects. Jpn J Appl Phys 44:8269. https://doi.org/10.1143/JJAP.44.8269

Herrmann J-M (1999) Heterogeneous photocatalysis: fundamentals and applications to the removal of various types of aqueous pollutants. Catal Today. 53:115-129. https://doi.org/10.1016/S09205861(99)00107-8

Hiragond CB, Khanna PK, More PV (2018) Probing the real-time photocatalytic activity of CdS QDs sensitized conducting polymers: featured PTh, PPy and PANI. Vacuum 155:159-168. https://doi. org/10.1016/j.vacuum.2018.06.009

Huo C, Dar AA, Nawaz A et al (2021) Groundwater contamination with the threat of COVID-19: insights into CSR theory of Carroll's pyramid. J King Saud Univ 33:101295. https://doi.org/10. 1016/j.jksus.2020.101295

Hussain S, Aneggi E, Goi D (2021) Catalytic activity of metals in heterogeneous Fenton-like oxidation of wastewater contaminants: a review. Environ Chem Lett. https://doi.org/10.1007/ s10311-021-01185-Z

Iervolino G, Zammit I, Vaiano V, Rizzo L (2020) Limitations and prospects for wastewater treatment by UV and visible-light-active heterogeneous photocatalysis: a critical review. Heterog Photocatal. https://doi.org/10.1007/978-3-030-49492-6_7

Jadoun S, Riaz U (2020) A review on the chemical and electrochemical copolymerization of conducting monomers: recent advancements and future prospects. Polym Technol Mater 59:484-504. https:// doi.org/10.1080/25740881.2019.1669647

Jadoun S, Ashraf SM, Riaz U (2017a) Tuning the spectral, thermal and fluorescent properties of conjugated polymers: via random copolymerization of hole transporting monomers. RSC Adv 7:32757-32768. https://doi.org/10.1039/c7ra04662f

Jadoun S, Sharma V, Ashraf SM, Riaz U (2017b) Sonolytic doping of poly(1-naphthylamine) with luminol: influence on spectral, morphological and fluorescent characteristics. Colloid Polym Sci. https://doi.org/10.1007/s00396-017-4055-3

Jadoun S, Verma A, Ashraf SM, Riaz U (2017c) A short review on the synthesis, characterization, and application studies of poly(1naphthylamine): a seldom explored polyaniline derivative. Colloid Polym Sci 295:1443-1453. https://doi.org/10.1007/ s00396-017-4129-2

Jadoun S, Biswal L, Riaz U (2018) Tuning the optical properties of poly(o-phenylenediamine-co-pyrrole) via template mediated copolymerization. Des Monomers Polym 21:75-81. https://doi. org/10.1080/15685551.2018.1459078

Jadoun S, Ashraf SM, Riaz U (2021) Insights into the spectral, thermal and morphological effects of co-oligomerization of pyrrole with luminol: a comparative experimental and computational study. Mater Sci Eng B. 273:115396. https://doi.org/10.1016/j.mseb. 2021.115396

Jadoun S, Rathore DS, Riaz U, Chauhan NPS (2021b) Tailoring of conducting polymers via copolymerization-a review. Eur Polym J 155:110561. https://doi.org/10.1016/j.eurpolymj.2021.110561
Jadoun S, Riaz U, Budhiraja V (2021) Biodegradable conducting polymeric materials for biomedical applications: a review. Med Dev Sens. 4:e10141. https://doi.org/10.1002/mds3.10141

Jadoun S, Riaz U, Yáñez J, Pal Singh Chauhan N (2021) Synthesis, characterization and potential applications of Poly(o-phenylenediamine) based copolymers and nanocomposites: a comprehensive review. Eur Polym J. 156:110600. https://doi.org/10.1016/j. eurpolymj.2021.110600

Jangid NK, Jadoun S, Kaur N (2020a) A Review on high-throughput synthesis, deposition of thin films and properties of polyaniline. Eur Polym J 125:109485. https://doi.org/10.1016/j.eurpolymj. 2020.109485

Jangid NK, Jadoun S, Yadav A et al (2020b) Polyaniline-TiO ${ }_{2}$-based photocatalysts for dyes degradation. Polym Bull. https://doi.org/ 10.1007/s00289-020-03318-w

Kanamarlapudi SLRK, Chintalpudi VK, Muddada S (2018) Application of biosorption for removal of heavy metals from wastewater. Biosorption. https://doi.org/10.5772/INTECHOPEN.77315

Katančić Z, Suka S, Vrbat K et al (2017) Synthesis of PEDOT/ZnO photocatalyst: validation of photocatalytic activity by degradation of Azo RR45 dye under solar and UV-A irradiation. Chem Biochem Eng Q. 31:385-394. https://doi.org/10.15255/CABEQ. 2017.1124

Ke J, Younis MA, Kong Y et al (2018) Nanostructured ternary metal tungstate-based photocatalysts for environmental purification and solar water splitting: a review. Nano-Micro Lett 10:1-27. https:// doi.org/10.1007/s40820-018-0222-4

Khan MI, Bhatti KA, Qindeel R et al (2017) Characterizations of multilayer $\mathrm{ZnO}$ thin films deposited by sol-gel spin coating technique. Results Phys 7:651-655. https://doi.org/10.1016/j.rinp.2016.12. 029

Khan F, Zahid M, Hanif MA, et al (2021) Photocatalytic polymeric composites for wastewater treatment. In: Aquananotechnology. Elsevier, pp 457-480. https://doi.org/10.1016/B978-0-12821141-0.00005-7

Khatamian M, Fazayeli M, Divband B (2014) Preparation, characterization and photocatalytic properties of polythiophene-sensitized zinc oxide hybrid nanocomposites. Mater Sci Semicond Process 26:540-547. https://doi.org/10.1016/j.mssp.2014.04.038

Khokhar D, Jadoun S, Arif R et al (2020) Copolymerization of o-phenylenediamine and 3-Amino-5-methylthio-1H-1,2,4-triazole for tuned optoelectronic properties and its antioxidant studies. J Mol Struct. https://doi.org/10.1016/j.molstruc.2020.129738

Khokhar D, Jadoun S, Arif R, Jabin S (2020b) Functionalization of conducting polymers and their applications in optoelectronics. Polym Technol Mater 60:465-487. https://doi.org/10.1080/25740 881.2020 .1819312

Khokhar D, Jadoun S, Arif R, Jabin S (2021) Tuning the spectral, thermal and morphological properties of Poly(o-phenylenediamineco-vaniline). Mater Res Innov. https://doi.org/10.1080/14328917. 2020.1870330

Kim H, Oh M-H, Yang BL (2020) Photocorrosion of polyaniline$\mathrm{ZnS}-\mathrm{ZnO}$ photoelectrode for water splitting. Thin Solid Films 693:137678. https://doi.org/10.1016/j.tsf.2019.137678

Kumar A, Billa S, Chaudhary S et al (2018) Ternary nanocomposite for solar light photocatalyic degradation of methyl orange. Inorg Chem Commun 97:191-195. https://doi.org/10.1016/j.inoche. 2018.09.038

Kumar L, Ragunathan V, Chugh M, Bharadvaja N (2021) Nanomaterials for remediation of contaminants: a review. Environ Chem Lett. https://doi.org/10.1007/s10311-021-01212-z

Lahrich S, Laghrib F, Farahi A et al (2021) Review on the contamination of wastewater by COVID-19 virus: impact and treatment. Sci Total Environ 751:142325. https://doi.org/10.1016/j.scito tenv. 2020.142325 
Li Z, Meng X, Zhang Z (2018) Recent development on MoS2-based photocatalysis: A review. J Photochem Photobiol C Photochem Rev 35:39-55. https://doi.org/10.1016/j.jphotochemrev.2017. 12.002

Luo J, Zhang S, Sun M et al (2019) A critical review on energy conversion and environmental remediation of photocatalysts with remodeling crystal lattice, surface, and interface. ACS Nano 13:9811-9840. https://doi.org/10.1021/acsnano.9b03649

Madima N, Mishra SB, Inamuddin I, Mishra AK (2020) Carbonbased nanomaterials for remediation of organic and inorganic pollutants from wastewater. A Review Environ Chem Lett 18:1169-1191. https://doi.org/10.1007/s10311-020-01001-0

Miranda C, Santander P, Matschullat J et al (2016) Degradation of organoarsenicals by heterogeneous photocatalysis using $\mathrm{ZnO}$, TiO2 and UVA. J Adv Oxid Technol 19:276-283. https://doi. org/10.1515/jaots-2016-0211

Mitra M, Ghosh A, Mondal A et al (2017) Facile synthesis of aluminium doped zinc oxide-polyaniline hybrids for photoluminescence and enhanced visible-light assisted photo-degradation of organic contaminants. Appl Surf Sci 402:418-428. https:// doi.org/10.1016/j.apsusc.2017.01.072

Mittal H, Kumar A, Khanuja M (2019) In-situ oxidative polymerization of aniline on hydrothermally synthesized MoSe2 for enhanced photocatalytic degradation of organic dyes. J Saudi Chem Soc 23:836-845. https://doi.org/10.1016/j.jscs.2019.02. 004

Mohammed AM, Mohtar SS, Aziz F et al (2021) $\mathrm{Cu}_{2} \mathrm{O} / \mathrm{ZnO}-\mathrm{PANI}$ ternary nanocomposite as an efficient photocatalyst for the photodegradation of Congo Red dye. J Environ Chem Eng 9:105065. https://doi.org/10.1016/j.jece.2021.105065

Mozafari M, Chauhan NPS (2019) Fundamentals and emerging applications of polyaniline. Elsevier

Nakata K, Fujishima A (2012) $\mathrm{TiO}_{2}$ photocatalysis: design and applications. J Photochem Photobiol C Photochem Rev 13:169-189. https://doi.org/10.1016/j.jphotochemrev.2012.06.001

Olad ALI, Behboudi S, Entezami ALIA (2012) Preparation, characterization and photocatalytic activity of $\mathrm{TiO} 2 /$ polyaniline core-shell nanocomposite. Bull Mater Sci 35:801-809. https://doi.org/10. 1007/s12034-012-0358-7

Olgun U, Gülfen M (2014) Doping of poly (o-phenylenediamine): spectroscopy, voltammetry, conductivity and band gap energy. React Funct Polym 77:23-29. https://doi.org/10.1016/j.reactfunct polym.2014.02.006

Organization WH (2020) Water, sanitation, hygiene and waste management for COVID-19: technical brief, 03 March 2020. World Health Organization

Ovando-Medina VM, López RG, Castillo-Reyes BE et al (2015) Composite of acicular rod-like $\mathrm{ZnO}$ nanoparticles and semiconducting polypyrrole photoactive under visible light irradiation for methylene blue dye photodegradation. Colloid Polym Sci 293:3459-3469. https://doi.org/10.1007/s00396-015-3717-2

Palominos R, Freer J, Mondaca MA, Mansilla HD (2008) Evidence for hole participation during the photocatalytic oxidation of the antibiotic flumequine. J Photochem Photobiol A Chem 193:139-145. https://doi.org/10.1016/j.jphotochem.2007.06.017

Patil SL, Pawar SG, Chougule MA et al (2012) Structural, morphological, optical, and electrical properties of PANI-ZnO nanocomposites. Int J Polym Mater Polym Biomater 61:809-820. https://doi. org/10.1080/00914037.2011.610051

Patil P, Gaikwad G, Patil DR, Naik J (2016) Synthesis of 1-D ZnO nanorods and polypyrrole/1-D $\mathrm{ZnO}$ nanocomposites for photocatalysis and gas sensor applications. Bull Mater Sci 39:655-665. https://doi.org/10.1007/s12034-016-1208-9

Pei Z, Ding L, Lu M et al (2014) Synergistic effect in polyanilinehybrid defective $\mathrm{ZnO}$ with enhanced photocatalytic activity and stability. J Phys Chem C 118:9570-9577. https://doi.org/10.1021/ jp5020143

Peng Y-G, Ji J-L, Zhang Y-L et al (2014) Preparation of poly(m-phenylenediamine)/ZnO composites and their photocatalytic activities for degradation of C.I. acid red 249 under UV and visible light irradiations. Environ Prog Sustain Energy 33:123-130. https://doi.org/10.1002/ep.11764

Podasca VE, Buruiana T, Buruiana EC (2019) Photocatalytic degradation of rhodamine $\mathrm{B}$ dye by polymeric films containing $\mathrm{ZnO}, \mathrm{Ag}$ nanoparticles and polypyrrole. J Photochem Photobiol A Chem 371:188-195. https://doi.org/10.1016/j.jphotochem.2018.11.016

Ponnamma D, Cabibihan J-J, Rajan M et al (2019) Synthesis, optimization and applications of $\mathrm{ZnO} /$ polymer nanocomposites. Mater Sci Eng C 98:1210-1240. https://doi.org/10.1016/j.msec.2019. 01.081

Ponnuchamy M, Kapoor A, Senthil Kumar P et al (2021) Sustainable adsorbents for the removal of pesticides from water: a review. Environ Chem Lett. https://doi.org/10.1007/s10311-021-01183-1

Pradeeba SJ, Sampath K (2019) Synthesis and characterization of Poly(azomethine)/ZnO nanocomposite toward photocatalytic degradation of methylene blue, malachite green, and bismarck brown. J Dyn Syst Meas Control. https://doi.org/10.1115/1.40420 90

Pradenas M, Yáñez J, Ranganathan S et al (2019) Multivariate approach to hydrogenated $\mathrm{TiO} 2$ photocatalytic activity under visible light. Water Environ Res 91:157-164. https://doi.org/10. 1002/wer.1014

Ramesan MT, Nidhisha V, Jayakrishnan P (2017) Synthesis, characterization and conducting properties of novel poly (vinyl cinnamate)/ zinc oxide nanocomposites via in situ polymerization. Mater Sci Semicond Process 63:253-260. https://doi.org/10.1016/j.mssp. 2017.02.027

Riaz U, Ashraf SM (2011) Semi-conducting poly(1-naphthylamine) nanotubes: a $\mathrm{pH}$ independent adsorbent of sulphonate dyes. Chem Eng J 174:546-555. https://doi.org/10.1016/j.cej.2011. 09.036

Riaz U, Ashraf SM (2012) Latent photocatalytic behavior of semi-conducting poly(1-naphthylamine) nanotubes in the degradation of Comassie Brilliant Blue RG-250. Sep Purif Technol 95:97-102. https://doi.org/10.1016/j.seppur.2012.04.029

Riaz U, Ashraf SM, Kashyap J (2015) Enhancement of photocatalytic properties of transitional metal oxides using conducting polymers: a mini review. Mater Res Bull 71:75-90. https://doi.org/ 10.1016/j.materresbull.2015.06.035

Riaz U, Ashraf SM, Budhiraja V et al (2016) Comparative studies of the photocatalytic and microwave-assisted degradation of alizarin red using $\mathrm{ZnO} /$ poly (1-naphthylamine) nanohybrids. J Mol Liq 216:259-267. https://doi.org/10.1016/j.molliq.2016.01.018

Riaz U, Jadoun S, Kumar P et al (2017) Influence of Luminol doping of poly(o-phenylenediamine) on the spectral, morphological and fluorescent properties: a potential biomarker for Leishmania parasite. ACS Appl Mater Interfaces acsami. https://doi.org/10. 1021/acsami.7b10325

Riaz U, Jadoun S, Kumar P et al (2018) Microwave-assisted facile synthesis of poly(luminol-co-phenylenediamine) copolymers and their potential application in biomedical imaging. RSC Adv. https://doi.org/10.1039/c8ra08373h

Riaz U, Ashraf SM, Jadoun S et al (2019) Spectroscopic and biophysical interaction studies of water-soluble dye modified poly (o-phenylenediamine) for its potential application in BSA detection and bioimaging. Sci Rep 9:8544. https://doi.org/10.1038/ s41598-019-44910-z

Rostami H, Omrani A, Rostami AA, Emamgholizadeh A (2015) Electrooxidation of hydrazine in alkaline medium at carbon paste electrode decorated with poly(P-phenylendiamine/ZnO) 
nanocomposite. Ionics (kiel) 21:1073-1080. https://doi.org/10. 1007/s11581-014-1279-9

Rueda-Marquez JJ, Levchuk I, Ibañez PF, Sillanpää M (2020) A critical review on application of photocatalysis for toxicity reduction of real wastewaters. J Clean Prod 258:120694. https://doi.org/10. 1016/j.jclepro.2020.120694

Sandoval C, Ranganathan S, Ramírez E et al (2019) Visible light assisted photodegradation of thimerosal by high performance $\mathrm{ZnFe}_{2} \mathrm{O}_{4}$ /poly (o-phenylenediamine) composite. Mater Res Bull 116:8-15. https://doi.org/10.1016/j.materresbull.2019.04.008

Saravanan R, Sacari E, Gracia F et al (2016) Conducting PANI stimulated $\mathrm{ZnO}$ system for visible light photocatalytic degradation of coloured dyes. J Mol Liq 221:1029-1033. https://doi.org/10. 1016/j.molliq.2016.06.074

Saravanan R, Gracia F, Stephen A (2017) Basic principles, mechanism, and challenges of photocatalysis bt - nanocomposites for visible light-induced photocatalysis. In: Pradhan D, Sohn Y (eds) Khan MM. Springer International Publishing

Sharma K (2020) Tuning the morphology of the $\mathrm{ZnO}$ nanostructures by seeded-growth method. Mater Today Proc 37:2971-2973. https:// doi.org/10.1016/j.matpr.2020.08.708

Sharma S, Singh S, Khare N (2016) Enhanced photosensitization of zinc oxide nanorods using polyaniline for efficient photocatalytic and photoelectrochemical water splitting. Int J Hydrogen Energy. 41:21088-21098. https://doi.org/10.1016/j.ijhydene.2016.08.131

Sharma VK, Jinadatha C, Lichtfouse E (2020) Environmental chemistry is most relevant to study coronavirus pandemics. Environ Chem Lett 18:993-996. https://doi.org/10.1007/s10311-020-01017-6

Sharma V, Maivizhikannan V, Rao VN et al (2021) Sea urchin shaped $\mathrm{ZnO}$ coupled with $\mathrm{MoS}_{2}$ and polyaniline as highly efficient photocatalysts for organic pollutant decomposition and hydrogen evolution. Ceram Int 47:10301-10313. https://doi.org/10.1016/j. ceramint.2020.09.199

Sibel ZOR, Budak B (2020) Investigation of the effect of PAn and PAn/ $\mathrm{ZnO}$ photocatalysts on $100 \%$ degradation of Congo red under UV visible light irradiation and lightless environment. Turkish $\mathbf{J}$ Chem 44:486-501. https://doi.org/10.3906/kim-1907-30

Silvestri S, Ferreira CD, Oliveira V et al (2019) Synthesis of PPy$\mathrm{ZnO}$ composite used as photocatalyst for the degradation of diclofenac under simulated solar irradiation. J Photochem Photobiol A Chem. 375:261-269. https://doi.org/10.1016/j.jphot ochem.2019.02.034

Sivakumar K, Senthil Kumar V, Haldorai Y (2012) Zinc oxide nanoparticles reinforced conducting poly(aniline-co-p-phenylenediamine) nanocomposite. Compos Interfaces 19:397-409. https:// doi.org/10.1080/15685543.2012.739502

Sivakumar K, Senthil Kumar V, Shim J-J, Haldorai Y (2014) Conducting copolymer/ZnO Nanocomposite: synthesis, characterization, and its photocatalytic activity for the removal of pollutants synth react inorganic. Met Nano-Metal Chem 44:1414-1420. https:// doi.org/10.1080/15533174.2013.809743

Sivakumar K, Senthil Kumar V, Shim J-J, Haldorai Y (2015) Poly(aniline-co-o-toluidine) encapsulated zinc oxide nanocomposite: preparation, characterization, and photocatalytic reduction of Cr(VI). Synth React Inorganic, Met Nano-Metal Chem 45:660-666. https://doi.org/10.1080/15533174.2014.965112
Subudhi S, Tripathy SP, Parida K (2021) Highlights of the characterization techniques on inorganic, organic (COF) and hybrid (MOF) photocatalytic semiconductors. Catal Sci Technol 11:392-415. https://doi.org/10.1039/D0CY02034F

Suresh R, Sandoval C, Ramírez E et al (2018) Solid-state synthesis and characterization of $\alpha-\mathrm{Fe}_{2} \mathrm{O}_{3} @ \mathrm{ZnO}$ nanocomposites with enhanced visible light driven photocatalytic activity. J Mater Sci Mater Electron 29:20347-20355. https://doi.org/10.1007/ s10854-018-0170-2

Swager TM (2017) 50th anniversary perspective: Conducting/semiconducting conjugated polymers. A personal perspective on the past and the future. Macromolecules 50:4867-4886. https://doi. org/10.1021/acs.macromol.7b00582

Talaiekhozani A, Rezania S, Kim K-H et al (2020) Recent advances in photocatalytic removal of organic and inorganic pollutants in air. J Clean Prod 278:123895. https://doi.org/10.1016/j.jclepro. 2020.123895

Vishnu D, Dhandapani B, Kannappan Panchamoorthy G et al (2021) Comparison of surface-engineered superparamagnetic nanosorbents with low-cost adsorbents of cellulose, zeolites and biochar for the removal of organic and inorganic pollutants: a review. Environ Chem Lett. https://doi.org/10.1007/s10311-021-01201-2

Yan B, Wang Y, Jiang X et al (2017) Flexible photocatalytic composite film of ZnO-Microrods/Polypyrrole. ACS Appl Mater Interfaces 9:29113-29119. https://doi.org/10.1021/acsami.7b08462

Yashni G, Al-Gheethi A, Mohamed R et al (2020) Photocatalysis of xenobiotic organic compounds in greywater using zinc oxide nanoparticles: a critical review. Water Environ J 35:190-217. https://doi.org/10.1111/wej.12619

Yu L, Chen W, Li D et al (2015) Inhibition of photocorrosion and photoactivity enhancement for $\mathrm{ZnO}$ via specific hollow $\mathrm{ZnO}$ core/ ZnS shell structure. Appl Catal B Environ 164:453-461. https:// doi.org/10.1016/j.apcatb.2014.09.055

Zhang C-L, Ma R-H, Yang M-R et al (2020) Synthesis, characterization, and photocatalytic performance of a ternary composite catalyst $\alpha$-SiW11Cr/PANI/ZnO. J Coord Chem 73:229-242. https:// doi.org/10.1080/00958972.2020.1727454

Zhao Y-P, Cai Z-S, Zhou Z-Y, Fu X-L (2011) Fabrication of conductive network formed by polyaniline- $\mathrm{ZnO}$ composite on fabric surfaces. Thin Solid Films. 519:5887-5891. https://doi.org/10. 1016/j.tsf.2011.02.088

Zhu J, Shao C, Li X et al (2018) Immobilization of ZnO/polyaniline heterojunction on electrospun polyacrylonitrile nanofibers and enhanced photocatalytic activity. Mater Chem Phys. 214:507515. https://doi.org/10.1016/j.matchemphys.2018.04.053

Zoshki A, Rahmani MB, Masdarolomoor F, Pilehrood SH (2019) Surface functionalization of PANI and PANI/ZnO hybrid nanofibers with metallic catalysts for ammonia sensing at room temperature. Mod Phys Lett B 33:1950175

Publisher's Note Springer Nature remains neutral with regard to jurisdictional claims in published maps and institutional affiliations. 